Supporting Information for:

\title{
The Formation and Fate of Carbonyls in Potable Water Reuse Systems
}

\author{
Emily L. Marron ${ }^{1,2}$, Carsten Prasse ${ }^{1,3}$, Jean Van Buren ${ }^{4,5}$, David L. Sedlak ${ }^{1,2^{*}}$ \\ ${ }^{1}$ Department of Civil and Environmental Engineering \\ University of California, Berkeley, California 94720 \\ ${ }^{2}$ NSF Engineering Research Center for Reinventing \\ the Nation's Urban Water Infrastructure (ReNUWIt) \\ ${ }^{3}$ Department of Environmental Health and Engineering \\ Johns Hopkins University, Baltimore, Maryland 21205 \\ ${ }^{4}$ Department of Chemistry \\ University of California, Berkeley, California 94720 \\ ${ }^{5}$ Department of Civil and Environmental Engineering \\ University of Southern California, Los Angeles, California 90089
}

Number of pages: 29; Figures: 12; Tables: 8

*corresponding author e-mail: sedlak@berkeley.edu 


\section{Table of Contents}

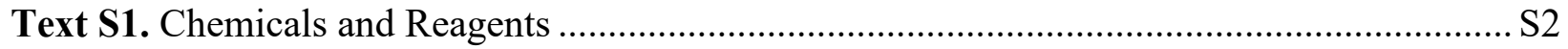

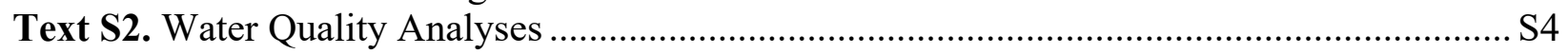

Text S3. Derivatization Method and Standard Addition Procedure ........................................ S8

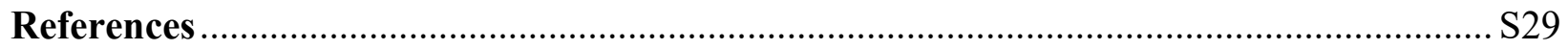

\section{List of Tables}

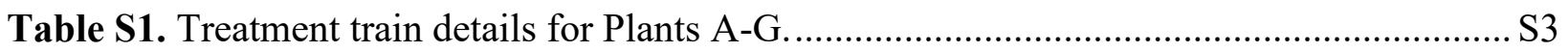

Table S2A-G. Water quality information for treatment Plants A-G.................................... S5

Table S3. Gradient method used for separation of the carbonyl compound mixture on HPLC. . S9

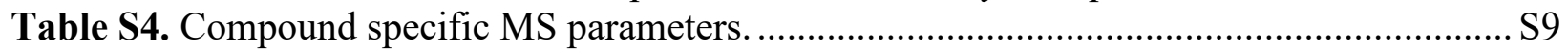

Tables S5A-G. Carbonyl compound concentrations in Plants A-G.................................... S13

Table S6. Chlorination experiment results compared to MFF samples .................................... S23

Table S7. Reaction rate constants $\left(k_{\mathrm{HO}} \cdot\right)$ between select carbonyl compounds and hydroxyl

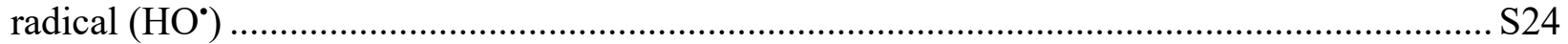

Table S8. Identified and unidentified peaks from TSH precursor ion scans (m/z 139) ............. S27

\section{List of Figures}

Figure S1. Derivatization of carbonyl compounds with p-toluenesulfonylhydrazine (TSH) ...... S8

Figure S2. Example chromatogram of carbonyl compound mixture..................................... S10

Figure S3. Peak areas for target carbonyl compounds as a function of increasing TSH

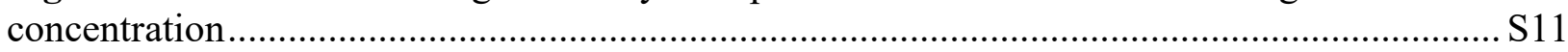

Figure S4. Example standard addition curves................................................................... S12

Figure S5. Formaldehyde contamination over time .......................................................... S12

Figure S6. Example chromatogram of TSH method for formaldehyde detection .................... S12

Figures S7A-G. Graphs of individual sampling events from 2018 and 2019 for Plants A-G... S20

Figure S8. Plant A precursor ion scan (m/z 139) for TSH-adducts .................................... S25

Figure S9. Plant D precursor ion scan (m/z 139) for TSH-adducts ....................................... S25

Figure S10. Plant E precursor ion scan $(\mathrm{m} / \mathrm{z} 139)$ for TSH-adducts........................................ S26

Figure S11. Plant F precursor ion scan $(\mathrm{m} / \mathrm{z} 139)$ for TSH-adducts..................................... S26

Figure S12. The contribution of carbonyl compounds and other chemicals to the total DOC

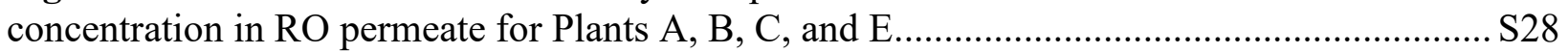




\section{Text S1. Chemicals and Reagents}

All chemicals and reagents were used as received, including: $p$-toluenesulfonylhydrazine (97\%), acetaldehyde ( $\geq 99 \%)$, acetone ( $\geq 99.5 \%$ ), acrolein ( $\geq 90 \%)$, propionaldehyde ( $\geq 98 \%)$, crotonaldehyde ( $\geq 99 \%$, predominantly trans), butyraldehyde ( $\geq 99.5 \%)$, trans-2-pentenal (95\%), 3-methyl crotonaldehyde (97\%), tiglic aldehyde ( $\geq 96 \%)$, pentanal ( $\geq 97.5 \%)$, trans-2-hexenal $(98 \%)$, hexanal (98\%), trans-2-heptenal (97\%), and sodium hypochlorite (4.00-4.99\%) from Sigma-Aldrich; LC-MS grade water (LiChroSolv) from Millipore-Sigma; formaldehyde (37\%), methanol (>99.9\%), acetonitrile (>99.9\%), and acetic acid $(99.7 \%)$ from Fisher; and 2,4hexadienal (95\%) from Alfa Aesar. 
Table S1. Treatment train details for Plants A-G. Plants A-F treated wastewater effluent, Plant G treated surface water. "O $\mathrm{O}_{3}$ " = ozone; "MF" = microfiltration; "UF" = ultrafiltration; "BAC" = biologically activated carbon filtration; "GAC" = granular activated carbon filtration; "RO" = reverse osmosis; "UV/ $\mathrm{H}_{2} \mathrm{O}_{2}$ " = advanced oxidation with UV light combined with hydrogen peroxide; "UV/HOCl" = advanced oxidation with UV light combined with hypochlorous acid; "UV" = disinfection with UV light.

\begin{tabular}{|c|c|c|c|c|}
\hline \multirow{2}{*}{$\begin{array}{l}\text { Treatment } \\
\text { Facility }\end{array}$} & \multirow{2}{*}{$\begin{array}{c}\text { Design } \\
\text { Capacity, } \\
\mathbf{m}^{3} \mathbf{s}^{-1} \\
\left(\mathbf{M G D}^{\mathbf{a}}\right)\end{array}$} & \multirow[b]{2}{*}{ Treatment Train } & \multicolumn{2}{|c|}{ Operational Parameters } \\
\hline & & & $\begin{array}{c}\mathrm{O}_{3} \\
\text { dose } \\
(\mathrm{mg} / \mathrm{L})\end{array}$ & AOP/Disinfection \\
\hline Plant A & $\begin{array}{l}8.8 \times 10^{-4} \\
(0.020)\end{array}$ & $\mathrm{O}_{3}-\mathrm{MF}-\mathrm{RO}-\mathrm{UV} / \mathrm{H}_{2} \mathrm{O}_{2}$ & $7-13$ & $\begin{array}{c}\mathrm{UV} \text { dose }=2,500 \mathrm{~mJ} / \mathrm{cm}^{2}, \\
{\left[\mathrm{H}_{2} \mathrm{O}_{2}\right]_{0}=5 \mathrm{mg} / \mathrm{L}}\end{array}$ \\
\hline Plant B & $\begin{array}{r}2.9 \\
(66)\end{array}$ & $\mathrm{O}_{3}-\mathrm{MF}-\mathrm{RO}-\mathrm{UV} / \mathrm{H}_{2} \mathrm{O}_{2}$ & 4 & $\begin{array}{c}\mathrm{UV} \text { dose }=\mathrm{N} / \mathrm{A}^{\mathrm{b}} \\
{\left[\mathrm{H}_{2} \mathrm{O}_{2}\right]_{0}=3 \mathrm{mg} / \mathrm{L}}\end{array}$ \\
\hline Plant C & $\begin{array}{c}0.0063 \\
(0.14)\end{array}$ & $\mathrm{MF}-\mathrm{RO}-\mathrm{UV} / \mathrm{H}_{2} \mathrm{O}_{2}$ & N.A. & $\begin{array}{c}\text { UV dose }=1,000 \mathrm{~mJ} / \mathrm{cm}^{2}, \\
{\left[\mathrm{H}_{2} \mathrm{O}_{2}\right]_{0}=2 \mathrm{mg} / \mathrm{L}}\end{array}$ \\
\hline Plant D & $\begin{array}{c}4.4 \\
(100)\end{array}$ & $\mathrm{MF}-\mathrm{RO}-\mathrm{UV} / \mathrm{H}_{2} \mathrm{O}_{2}$ & N.A. & $\begin{array}{c}\mathrm{UV} \text { dose }=300 \mathrm{~mJ} / \mathrm{cm}^{2} \\
{\left[\mathrm{H}_{2} \mathrm{O}_{2}\right]_{0}=3 \mathrm{mg} / \mathrm{L}}\end{array}$ \\
\hline Plant E & $\begin{array}{c}0.044 \\
(1)\end{array}$ & $\begin{array}{c}\mathrm{O}_{3}-\mathrm{BAC}-\mathrm{UF}-\mathrm{RO}- \\
\mathrm{UV} / \mathrm{HOCl}\end{array}$ & 7 & $\begin{array}{l}\mathrm{UV} \text { dose }=850 \mathrm{~mJ} / \mathrm{cm}^{2} \\
\mathrm{NaOCl} \text { dose }=1-3 \mathrm{mg} / \mathrm{L}\end{array}$ \\
\hline Plant F & $\begin{array}{l}0.044 \\
(1)\end{array}$ & $\begin{array}{c}\text { Settling }-\mathrm{O}_{3}-\mathrm{BAC}-\mathrm{GAC} \\
-\mathrm{UV}-\mathrm{HOCl}\end{array}$ & $5.1-6.3$ & $\begin{array}{l}\mathrm{UV} \text { dose }=186 \mathrm{~mJ} / \mathrm{cm}^{2} \\
\mathrm{NaOCl} \text { dose }=3 \mathrm{mg} / \mathrm{L}\end{array}$ \\
\hline Plant $\mathrm{G}$ & $\begin{array}{c}1.0 \\
(24)\end{array}$ & $\begin{array}{c}\text { Flocculation - } \\
\text { Sedimentation }-\mathrm{O}_{3}- \\
\text { Filtration }^{\mathrm{c}}-\mathrm{NH}_{2} \mathrm{Cl}\end{array}$ & $1.5-2$ & $\mathrm{NH}_{2} \mathrm{Cl}$ dose $=2 \mathrm{mg} / \mathrm{L}$ \\
\hline
\end{tabular}

${ }^{\mathrm{a}} \mathrm{MGD}=$ million gallons per day.

${ }^{b} \mathrm{~N} / \mathrm{A}=$ not available; but installed with a sufficient dose to operate as a $\mathrm{UV} / \mathrm{H}_{2} \mathrm{O}_{2}$ for control of trace organics in RO-treated water.

${ }^{\mathrm{c}}$ Filtration=dual media anthracite/sand filters. 


\section{Text S2. Water Quality Analyses}

The $\mathrm{pH}$ of water samples was measured using an Oakton $700 \mathrm{pH}$ probe (Tables S2A-G).

Full spectral scans (200-500 nm) of each sample were taken on an Agilent Cary $60 \mathrm{UV}$-vis spectrophotometer. UV absorbance at $254 \mathrm{~nm}\left(\mathrm{UV}_{254}\right)$ is reported in Tables S2A-G.

Dissolved organic carbon (DOC) values reported were measured (as non-purgeable organic carbon) using a Shimadzu TOC-V total organic carbon analyzer (instrument detection limit 0.2 mg C/L). ${ }^{1}$ Plants A-G had online total organic carbon (TOC) analyzers with real-time measurements. Plants A-E had online TOC analyzers reading reverse osmosis permeate; Plant F had online TOC analyzers for the secondary effluent, post-settling, the biologically active filtration effluent, and in the final product water. TOC measurements displayed on the analyzers were recorded at the time of sampling. All TOC data are recorded in Tables S2A-G. 
Table S2A-G. Water quality information for treatment Plants A-G.

Table S2A. Water quality information for treatment Plant A samples. Post-NaOCl samples were collected during the 2019 event only ( $\mathrm{NaOCl}$ dose was approximately $6 \mathrm{mg} / \mathrm{L}$ ). Ozone dose was $13 \mathrm{~g} / \mathrm{L}$ in 2018 and $7 \mathrm{mg} / \mathrm{L}$ in 2019.

\begin{tabular}{|c|c|c|c|c|c|c|}
\hline 2018 & Sec Eff & Post-NaOCl & Post-Ozone & MF Effluent & RO Permeate & Post-UV/ $\mathrm{H}_{2} \mathrm{O}_{2}$ \\
\hline $\mathrm{pH}$ & 6.91 & n.a. & 6.98 & 6.93 & 5.11 & 5.03 \\
\hline $\mathrm{UV}_{254}$ & 0.197 & n.a. & 0.157 & 0.145 & 0.004 & 0.002 \\
\hline $\mathrm{DOC}$ (mg C/L) & 13.41 & n.a. & 13.6 & 11.72 & 0.21 & $<0.2$ \\
\hline Online TOC (mg/L) & & & & & 0.167 & \\
\hline 2019 & Sec Eff & Post-NaOCl & Post-Ozone & MF Effluent & RO Permeate & Post-UV/ $\mathrm{H}_{2} \mathrm{O}_{2}$ \\
\hline $\mathrm{pH}$ & 7.78 & 7.76 & 7.88 & 7.9 & 5.3 & 5.65 \\
\hline$U_{254}$ & 0.276 & 0.224 & 0.216 & 0.171 & 0.004 & 0.002 \\
\hline $\mathrm{DOC}(\mathrm{mg} \mathrm{C} / \mathrm{L})$ & 16.24 & 15.81 & 14.75 & 13.69 & 0.29 & 0.20 \\
\hline Online TOC $(\mathrm{mg} / \mathrm{L})$ & & & & & 0.192 & \\
\hline
\end{tabular}

Table S2B. Water quality information for treatment Plant B samples. Ozone dose was $4 \mathrm{mg} / \mathrm{L}$ in 2018 and 2019. $\mathrm{NaOCl}$ dose (added after ozone) was $6 \mathrm{mg} / \mathrm{L}$.

\begin{tabular}{llllll}
\hline 2018 & Sec Eff & Post-Ozone & MF Effluent & RO Permeate & Post-UV/H $\mathrm{H}_{2} \mathrm{O}_{2}$ \\
\hline $\mathrm{pH}$ & 7.11 & 6.81 & 7.19 & 5.84 & 5.79 \\
$\mathrm{UV}_{254}$ & 0.265 & 0.270 & 0.183 & 0.126 & 0.004 \\
$\mathrm{DOC}(\mathrm{mg} \mathrm{C} / \mathrm{L})$ & 19.14 & 18.91 & 14.68 & 0.24 & 0.25 \\
Online TOC $(\mathrm{mg} / \mathrm{L})$ & & & & 0.190 & \\
\hline
\end{tabular}

\begin{tabular}{llllll}
\hline 2019 & Sec Eff & Post-Ozone & MF Effluent & RO Permeate & Post- $\mathrm{UV} / \mathrm{H}_{2} \mathrm{O}_{2}$ \\
\hline $\mathrm{pH}$ & 7.59 & 7.36 & 8.04 & 5.88 & 5.89 \\
$\mathrm{UV}_{254}$ & 0.344 & 0.333 & 0.184 & 0.009 & 0.002 \\
$\mathrm{DOC}(\mathrm{mg} \mathrm{C} / \mathrm{L})$ & 18.79 & 18.23 & 15.24 & $<0.2$ & $<0.2$ \\
Online TOC $(\mathrm{mg} / \mathrm{L})$ & & & & 0.180 & \\
\hline
\end{tabular}


Table S2C. Water quality information for treatment Plant $\mathrm{C}$ samples. $\mathrm{NaOCl}$ dose (added prior to $\mathrm{MF}$ ) was $12 \mathrm{mg} / \mathrm{L}$ in 2018 and $9.8 \mathrm{mg} / \mathrm{L}$ in 2019.

\begin{tabular}{|c|c|c|c|c|c|}
\hline 2018 & Sec Eff & MF Feed & MF Effluent & RO Permeate & Post-UV/ $\mathrm{H}_{2} \mathrm{O}_{2}$ \\
\hline $\mathrm{pH}$ & 7.19 & 7.14 & 7.28 & 5.49 & 5.64 \\
\hline $\mathrm{UV}_{254}$ & 0.128 & 0.120 & 0.116 & 0.006 & 0.005 \\
\hline $\mathrm{DOC}(\mathrm{mg} \mathrm{C} / \mathrm{L})$ & 7.36 & 7.46 & 6.72 & $<0.2$ & $<0.2$ \\
\hline Online TOC $(\mathrm{mg} / \mathrm{L})$ & & & & 0.130 & \\
\hline 2019 & Sec Eff & MF Feed & MF Effluent & RO Permeate & Post-UV/ $\mathrm{H}_{2} \mathrm{O}_{2}$ \\
\hline $\mathrm{pH}$ & 7.4 & 7.49 & 7.88 & 5.51 & 5.6 \\
\hline $\mathrm{UV}_{254}$ & 0.179 & 0.156 & 0.145 & 0.007 & 0.001 \\
\hline $\mathrm{DOC}(\mathrm{mg} \mathrm{C} / \mathrm{L})$ & 7.37 & 7.53 & 6.60 & $<0.2$ & $<0.2$ \\
\hline Online TOC $(\mathrm{mg} / \mathrm{L})$ & & & & 0.090 & \\
\hline
\end{tabular}

Table S2D. Water quality information for treatment Plant D samples. $\mathrm{NaOCl}$ dose (added prior to $\mathrm{MF}$ ) was $10.8 \mathrm{mg} / \mathrm{L}$ in $2018,8.5 \mathrm{mg} / \mathrm{L}$ in 2019.

\begin{tabular}{|c|c|c|c|c|c|}
\hline 2018 & Sec Eff & MF Feed & MF Effluent & RO Permeate & Post-UV/ $\mathrm{H}_{2} \mathrm{O}_{2}$ \\
\hline $\mathrm{pH}$ & 7.33 & 7.32 & 7.26 & 5.83 & 5.61 \\
\hline $\mathrm{UV}_{254}$ & 0.213 & 0.210 & 0.158 & 0.008 & 0.003 \\
\hline $\mathrm{DOC}(\mathrm{mg} \mathrm{C} / \mathrm{L})$ & 7.92 & 7.63 & 6.88 & $<0.2$ & $<0.2$ \\
\hline Online TOC (mg/L) & & & & 0.055 & \\
\hline 2019 & Sec Eff & MF Feed & MF Effluent & RO Permeate & Post-UV/ $\mathrm{H}_{2} \mathrm{O}_{2}$ \\
\hline $\mathrm{pH}$ & 7.84 & 7.66 & 7.45 & 5.93 & 6.33 \\
\hline $\mathrm{UV}_{254}$ & 0.238 & 0.230 & 0.190 & 0.009 & 0.004 \\
\hline $\mathrm{DOC}(\mathrm{mg} \mathrm{C} / \mathrm{L})$ & 7.92 & 7.84 & 6.21 & $<0.2$ & $<0.2$ \\
\hline Online TOC (mg/L) & & & & 0.062 & \\
\hline
\end{tabular}


Table S2E. Water quality information for treatment Plant E samples. Ozone dose was $7 \mathrm{mg} / \mathrm{L}$ in both 2018 and 2019.

\begin{tabular}{lllllll}
\hline 2018 & Tert Eff & Post-Ozone & $\begin{array}{l}\text { BAC } \\
\text { Effluent }\end{array}$ & UF Effluent & RO Permeate & Post-UV/HOCl \\
\hline $\mathrm{pH}$ & 7.22 & 6.66 & 6.84 & 6.69 & 5.20 & 5.98 \\
$\mathrm{UV}_{254}$ & 0.251 & 0.221 & 0.131 & 0.146 & 0.006 & 0.005 \\
$\begin{array}{l}\text { DOC (mg C/L) } \\
\begin{array}{l}\text { Online TOC } \\
\text { (mg/L) }\end{array}\end{array}$ & 7.295 & 7.232 & 4.896 & 4.89 & $<0.2$ & $<0.2$ \\
\hline
\end{tabular}

\begin{tabular}{lllllll}
\hline 2019 & Tert Eff & Post-Ozone & $\begin{array}{l}\text { BAC } \\
\text { Effluent }\end{array}$ & UF Effluent & RO Permeate & Post UV-HOCl \\
\hline $\mathrm{pH}$ & 7.34 & 7.11 & 6.93 & 6.93 & 5.55 & 5.86 \\
$\mathrm{UV}_{254}$ & 0.179 & 0.081 & 0.065 & 0.065 & 0.006 & 0.002 \\
$\begin{array}{l}\text { DOC }(\mathrm{mg} \mathrm{C} / \mathrm{L}) \\
\begin{array}{l}\text { Online TOC } \\
\text { (mg/L) }\end{array}\end{array}$ & 8.459 & 8.448 & 6.27 & 5.556 & $<0.2$ & $<0.2$ \\
\hline
\end{tabular}

Table S2F. Water quality information for treatment Plant F samples. Ozone dose was $6.3 \mathrm{mg} / \mathrm{L}$ in 2018 and $5.1 \mathrm{mg} / \mathrm{L}$ in 2019.

\begin{tabular}{|c|c|c|c|c|c|c|}
\hline 2018 & Sec Eff & $\begin{array}{l}\text { Post- } \\
\text { Settling }\end{array}$ & $\begin{array}{l}\text { Post- } \\
\text { Ozone }\end{array}$ & $\begin{array}{l}\text { BAF } \\
\text { Effluent }\end{array}$ & $\begin{array}{l}\text { GAC } \\
\text { Effluent }\end{array}$ & $\begin{array}{l}\text { Final } \\
\text { effluent }\end{array}$ \\
\hline $\mathrm{pH}$ & 7.17 & 7.42 & 6.74 & 6.86 & 6.78 & 7.6 \\
\hline $\mathrm{UV}_{254}$ & 0.307 & 0.148 & 0.069 & 0.053 & 0.027 & 0.019 \\
\hline $\mathrm{DOC}$ (mg C/L) & 8.58 & 7.04 & 6.82 & 5.04 & 2.67 & 2.56 \\
\hline Online TOC (mg/L) & 9.00 & 6.78 & & 4.94 & & 2.75 \\
\hline 2019 & Sec Eff & $\begin{array}{l}\text { Post- } \\
\text { Settling }\end{array}$ & $\begin{array}{l}\text { Post- } \\
\text { Ozone }\end{array}$ & $\begin{array}{l}\text { BAF } \\
\text { Effluent }\end{array}$ & $\begin{array}{l}\text { GAC } \\
\text { Effluent }\end{array}$ & $\begin{array}{l}\text { Final } \\
\text { effluent }\end{array}$ \\
\hline $\mathrm{pH}$ & 7.36 & 7.15 & 7.09 & 7.48 & 7.37 & 7.55 \\
\hline $\mathrm{UV}_{254}$ & 0.238 & 0.151 & 0.089 & 0.065 & 0.037 & 0.025 \\
\hline $\mathrm{DOC}(\mathrm{mg} \mathrm{C} / \mathrm{L})$ & 9.89 & 8.19 & 7.85 & 4.97 & 2.33 & 2.00 \\
\hline Online TOC (mg/L) & 9.10 & 7.55 & & 5.03 & 3.37 & 2.52 \\
\hline
\end{tabular}

Table S2G. Water quality information for treatment Plant G samples. Ozone dose was $1.5 \mathrm{mg} / \mathrm{L}$ in 2019-A, and $2 \mathrm{mg} / \mathrm{L}$ in 2019-B.

\begin{tabular}{llllll}
\hline 2019-A & Raw Water & Settled & Post-Ozone & Post-Filtration & Post-disinfection \\
\hline $\mathrm{pH}$ & 7.81 & 6.76 & 6.71 & 6.97 & 8.13 \\
$\mathrm{UV}_{254}$ & 0.169 & 0.055 & 0.025 & 0.025 & 0.041 \\
$\mathrm{DOC}(\mathrm{mg} \mathrm{C} / \mathrm{L})$ & 4.31 & 2.72 & 2.48 & 2.60 & 2.60 \\
\hline \multicolumn{7}{l}{} & & & & \\
\hline 2019-B & Raw Water & Settled & Post-Ozone & Post-Filtration & Post-disinfection \\
\hline $\mathrm{pH}$ & 7.79 & 6.85 & 7.01 & 6.95 & 7.99 \\
$\mathrm{UV}_{254}$ & 0.169 & 0.051 & 0.022 & 0.021 & 0.035 \\
$\mathrm{DOC}(\mathrm{mg} \mathrm{C/L})$ & 4.59 & n.d. & n.d. & n.d. & 3.04 \\
\hline
\end{tabular}




\section{Text S3. Derivatization Method and Standard Addition Procedure}

p-toluenesulfonylhydrazine (TSH) was added in excess for derivatization (Figure S1) with 13 target carbonyl compounds (i.e., acetaldehyde, acetone, acrolein, propionaldehyde, crotonaldehyde, butyraldehyde, 3-methylcrotonaldehyde, trans-2-pentenal, pentanal, 2,4hexadienal, trans-2-hexenal, hexanal, and trans-2-heptenal). Derivatized carbonyl compounds were analyzed using optimized parameters in LC-MS/MS (Tables S3-S4). Example chromatogram is shown in Figure S2.

Increasing amounts of TSH were added to a $100 \mu \mathrm{g} \mathrm{L}^{-1}$ aldehyde mixture to determine the optimal concentration for derivatization. On the basis of compound response curves (Figure S3), a concentration of $25 \mathrm{mg} / \mathrm{L}$ was selected and used thereafter, with the exception of formaldehyde, which was analyzed after addition of $10 \mathrm{mg} / \mathrm{L} \mathrm{TSH}$ to avoid contamination from laboratory air. Formaldehyde was derivatized separately from the other carbonyl compounds due to quantification difficulties stemming from laboratory and stock solution contamination (Figure S5). Due to background formaldehyde in the Milli-Q system, LC-MS grade water was used for all formaldehyde analyses. A shorter HPLC method was used for the formaldehyde analysis (isocratic method; $50 \% 0.1 \%$ acetic acid in LC-MS water, $50 \%$ methanol, flow rate $=0.5$ $\mathrm{mL} / \mathrm{min}$; example chromatogram Figure S6).

More consistent and reliable standard addition curves were obtained by preparing dilutions using a formaldehyde stock solution $(0.1 \mathrm{M})$ that had equilibrated in the refrigerator $\left(4{ }^{\circ} \mathrm{C}\right)$ for at least 12 hours prior to use. Using the formaldehyde reagent without this dilution step yielded less consistent results. The formaldehyde standard reagent contained methanol (10-15\%) as a stabilizer to prevent polymerization; therefore, equilibrium of the formaldehyde-methanol-water mixture could have interfered with derivatization. ${ }^{2}$

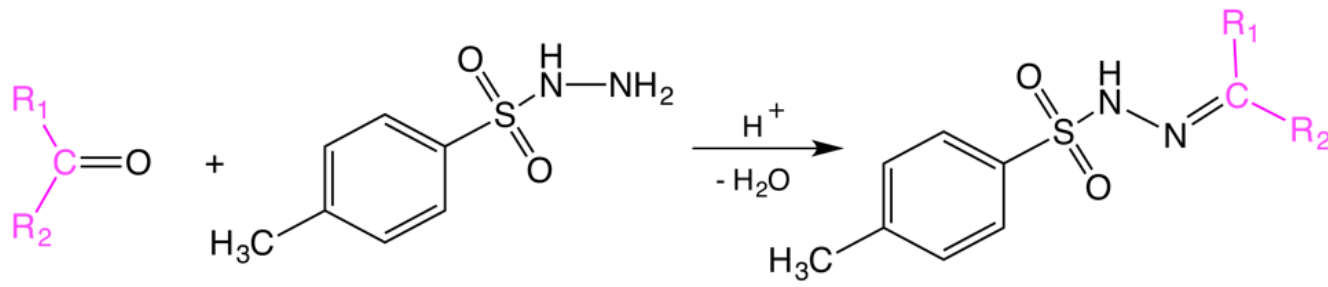

Figure S1. Derivatization of carbonyl compounds with p-toluenesulfonylhydrazine (TSH). For formaldehyde, $\mathrm{R}_{1}$ and $\mathrm{R}_{2}=\mathrm{H}$; for other aldehydes, $\mathrm{R}_{1}=\mathrm{H}, \mathrm{R}_{2}=\mathrm{C}_{2-6}$; for acetone, $\mathrm{R}_{1}$ and $\mathrm{R}_{2}=\mathrm{CH}_{3}$. 
Table S3. Gradient method used for separation of the carbonyl compound mixture on HPLC. $\mathrm{A}=0.1 \%$ acetic acid, $\mathrm{B}=$ methanol.

\begin{tabular}{|l|l|l|}
\hline Time $(\min )$ & $\mathrm{A}[\%]$ & $\mathrm{B}[\%]$ \\
\hline 0 & 60 & 40 \\
2 & 60 & 40 \\
5 & 40 & 60 \\
11 & 5 & 95 \\
16 & 5 & 95 \\
16.1 & 60 & 40 \\
21 & 60 & 40 \\
\hline
\end{tabular}

Table S4. Compound specific MS parameters. All compounds were analyzed in positive ionization mode, using a fragmentor voltage of $100 \mathrm{~V}$, and a cell accelerator voltage of $7 \mathrm{~V}$. Other parameters include: drying gas temperature of $350^{\circ} \mathrm{C}$, gas flow of $10 \mathrm{~L} \mathrm{~min}^{-1}$, a nozzle voltage of $300 \mathrm{~V}$, and a capillary voltage of $3600 \mathrm{~V}$.

\begin{tabular}{|l|l|l|l|l|}
\hline Compound & Retention Time & Precursor ion (amu) & Product ions (amu) & Collision energy (V)a \\
\hline TSH & 3.95 & 187 & $65,91,139$ & $40,20,20$ \\
\hline Formaldehyde & 5.94 & 199 & $77,91,105,139$ & $30,40,10,10$ \\
\hline Acetaldehyde & 6.71 & 213 & $57,93,139$ & $10,10,10$ \\
\hline Acrolein & 8.81 & 225 & $69,91,139$ & $20,30,20$ \\
\hline Acetone & 7.65 & 227 & $71,93,139$ & $10,40,10$ \\
\hline Propionaldehyde & 8.88 & 227 & $71,111,139$ & $10,10,10$ \\
\hline Crotonaldehyde & 9.78 & 239 & $69,83,139$ & $10,10,10$ \\
\hline Butyraldehyde & 10.11 & 241 & $86,85,139$ & $10,10,20$ \\
\hline Trans-2-pentenal & 10.9 & 253 & $69,97,139$ & $10,10,10$ \\
\hline 3-Methyl crotonaldehyde & 10.6 & 253 & $83,97,139$ & $20,10,10$ \\
\hline Pentanal & 11.2 & 255 & $82,99,139$ & $10,10,20$ \\
\hline 2,4-Hexadienal & 11.4 & 265 & $69,81,109,139$ & $20,20,10,20$ \\
\hline Trans-2-hexenal & 11.9 & 267 & $69,111,139$ & $10,10,10$ \\
\hline Hexanal & 12.1 & 269 & $113,139,157$ & $10,20,10$ \\
\hline Trans-2-heptenal & 12.7 & 281 & $69,125,139$ & $20,10,20$ \\
\hline
\end{tabular}

${ }^{\mathrm{a} C}$ Collision energies listed respectively for product ions in adjacent column. 


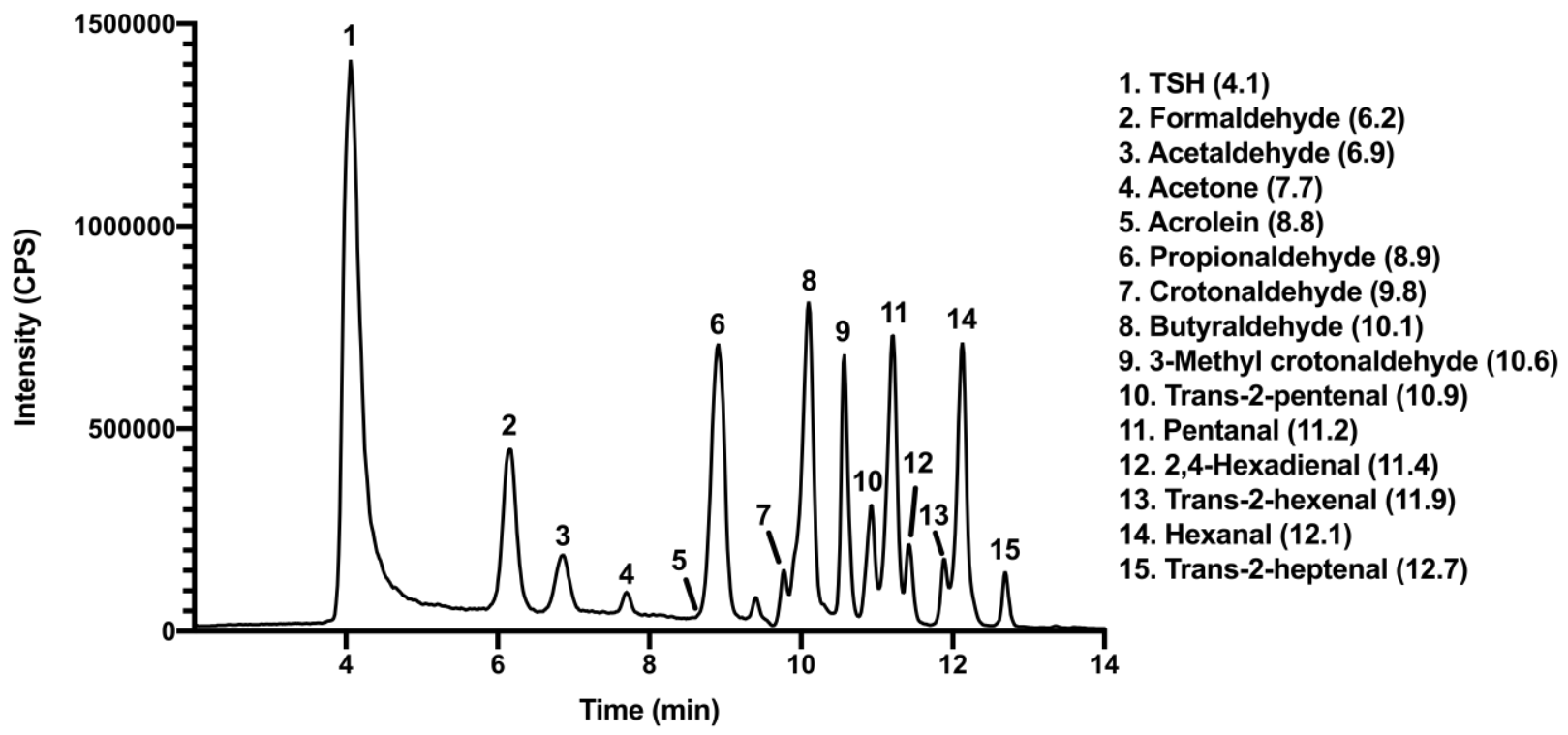

Figure S2. Example chromatogram of carbonyl compound mixture $(50 \mu \mathrm{g} / \mathrm{L})$ using the HPLC method from Table S1 (TSH = $25 \mathrm{mg} / \mathrm{L})$. Retention time (minutes) in parentheses. 

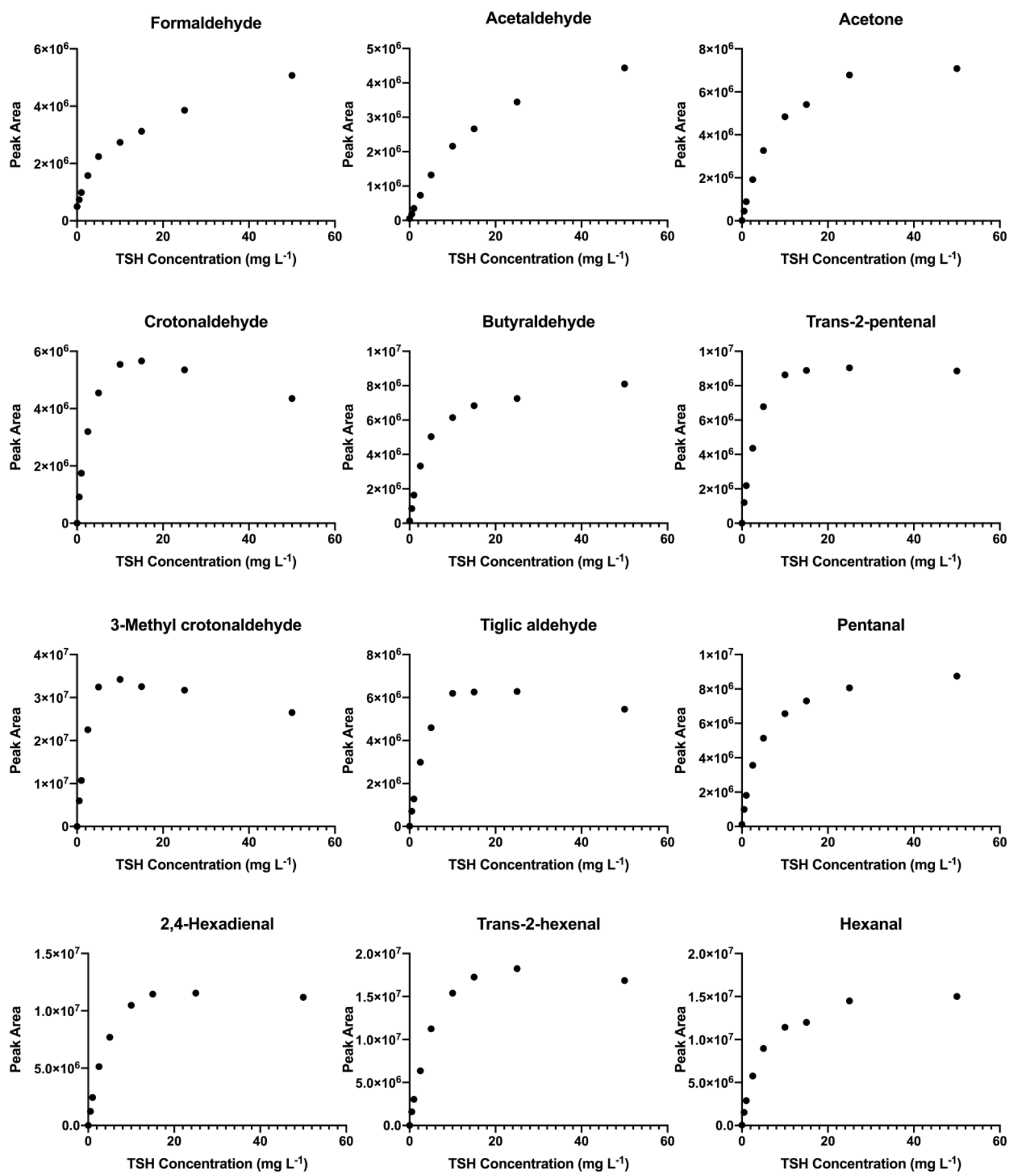

Figure S3. Peak areas for target carbonyl compounds as a function of increasing TSH concentration. Initial concentration of carbonyl compounds was $100 \mu \mathrm{g} \mathrm{L}^{-1}$. 
(a) formaldehyde

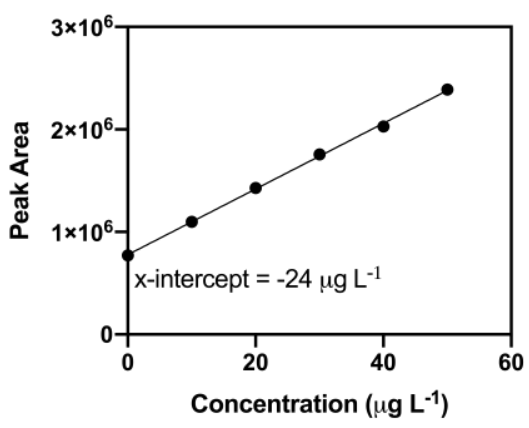

(b) acetone

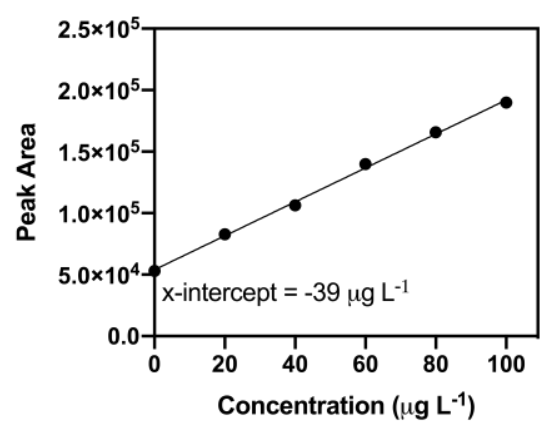

(c) acrolein

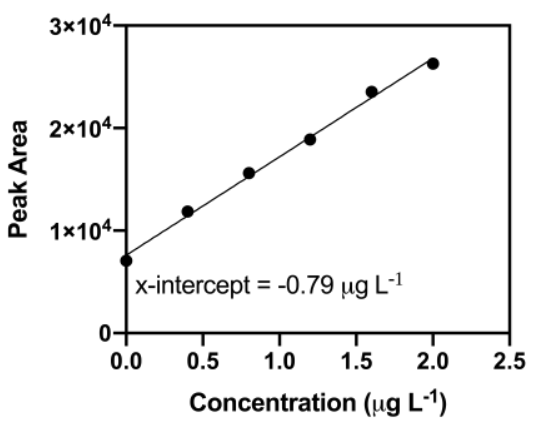

Figure S4. Example standard addition curves for (a) formaldehyde in a MF-feed sample from Plant D;(b) acetone in a post-ozone sample from Plant A; and, (c) acrolein in a RO permeate sample from Plant B.

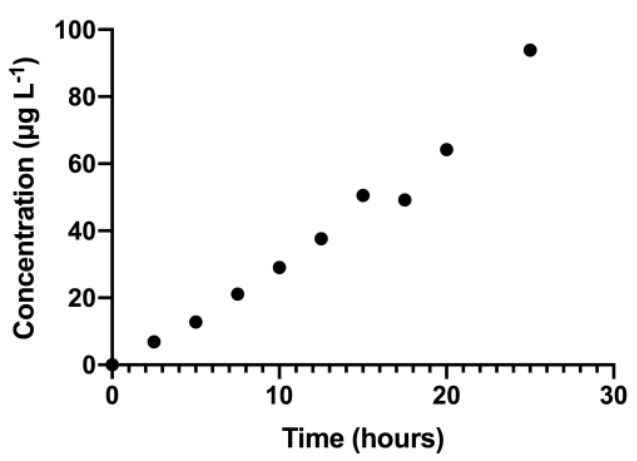

Figure S5. Formaldehyde contamination over time. Concentration of formaldehyde in a sample made from LC-MS water initially containing $0 \mu \mathrm{g} \mathrm{L}^{-1}$ of formaldehyde, as a function of time. Sample sat at room temperature in the autosampler tray of the HPLC-MS/MS for the duration of the experiment.

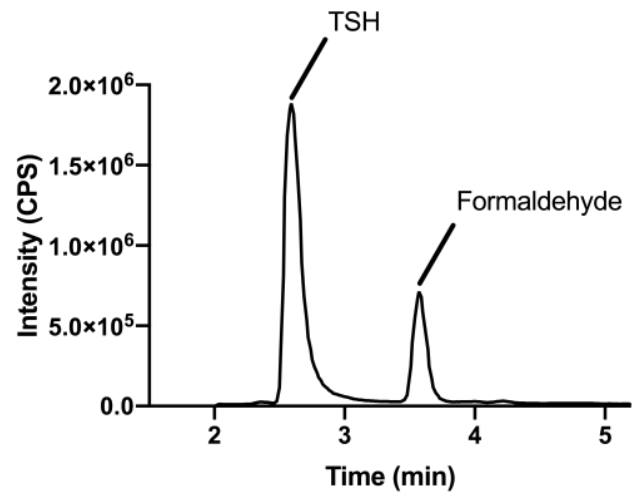

Figure S6. Example chromatogram of TSH method for formaldehyde detection. Peaks labeled for TSH (retention time, $\mathrm{RT}=2.59 \mathrm{~min}$ ) and $\mathrm{TSH}$-derivatized formaldehyde $(\mathrm{RT}=3.57 \mathrm{~min})$. 
Tables S5A-G. Carbonyl compound concentrations in Plants A-G

Table S5A. Concentrations ( $\mu \mathrm{g} \mathrm{L}^{-1}$ ) of carbonyl compounds in Plant A.

\begin{tabular}{|c|c|c|c|c|c|}
\hline Plant A - 2018 & WW Eff & $\mathrm{O}_{3} \mathrm{Eff}$ & MF Eff & $\begin{array}{l}\text { RO } \\
\text { Permeate }\end{array}$ & $\mathrm{UV} / \mathrm{H}_{2} \mathrm{O}_{2} \mathrm{Eff}$ \\
\hline Formaldehyde & 3.67 & 79.7 & 78.5 & 42.0 & 27.4 \\
\hline Acetaldehyde & 11.7 & 115 & 114 & 27.1 & 15.1 \\
\hline Acetone & 10.9 & 44.7 & 41.3 & 28.0 & 25.3 \\
\hline Propionaldehyde & 1.50 & 17.7 & 15.6 & 8.10 & 2.20 \\
\hline Butyraldehyde & 1.20 & 9.60 & 7.60 & 3.40 & 1.02 \\
\hline Pentanal & 1.35 & 11.5 & 9.10 & 3.10 & 0.975 \\
\hline Hexanal & 1.34 & 21.7 & 19.2 & 6.70 & 2.70 \\
\hline Acrolein & 0.300 & 4.23 & 4.48 & 2.37 & 0.320 \\
\hline Crotonaldehyde & $<0.010$ & 0.540 & 0.430 & 0.190 & 0.025 \\
\hline 3-methyl-crotonaldehyde & $<0.010$ & 0.460 & 0.430 & 0.190 & $<0.010$ \\
\hline Trans-2-pentenal & $<0.010$ & 0.180 & 0.179 & 0.055 & $<0.010$ \\
\hline 2,4-hexadienal & $<0.010$ & $<0.010$ & $<0.010$ & $<0.010$ & $<0.010$ \\
\hline Trans-2-hexenal & $<0.010$ & 0.098 & 0.089 & 0.030 & $<0.010$ \\
\hline Trans-2-heptenal & $<0.010$ & $<0.010$ & $<0.010$ & $<0.010$ & $<0.010$ \\
\hline Plant A - 2019 & WW Eff & $\mathrm{O}_{3} \mathrm{Eff}$ & MF Eff & $\begin{array}{l}\text { RO } \\
\text { Permeate }\end{array}$ & $\mathrm{UV} / \mathrm{H}_{2} \mathrm{O}_{2} \mathrm{Eff}$ \\
\hline Formaldehyde & 8.00 & 53.3 & 50.7 & 28.0 & 19.0 \\
\hline Acetaldehyde & 12.8 & 116 & 113 & 45.9 & 25.3 \\
\hline Acetone & 11.8 & 39.1 & 32.9 & 22.0 & 19.4 \\
\hline Propionaldehyde & 1.64 & 14.8 & 14.7 & 6.49 & 2.09 \\
\hline Butyraldehyde & 1.10 & 5.78 & 5.44 & 2.29 & 0.655 \\
\hline Pentanal & 0.910 & 7.43 & 6.69 & 2.27 & 0.721 \\
\hline Hexanal & 1.01 & 13.3 & 12.3 & 3.60 & 1.21 \\
\hline Acrolein & 0.200 & 3.11 & 3.4 & 1.89 & 0.150 \\
\hline Crotonaldehyde & $<0.010$ & 0.860 & 0.810 & 0.383 & 0.054 \\
\hline 3-methyl-crotonaldehyde & $<0.010$ & 0.340 & 0.290 & 0.098 & $<0.010$ \\
\hline Trans-2-pentenal & $<0.010$ & 0.220 & 0.170 & 0.068 & $<0.010$ \\
\hline 2,4-hexadienal & $<0.010$ & $<0.010$ & $<0.010$ & $<0.010$ & $<0.010$ \\
\hline Trans-2-hexenal & $<0.010$ & 0.123 & 0.109 & 0.033 & $<0.010$ \\
\hline Trans-2-heptenal & $<0.010$ & $<0.010$ & $<0.010$ & $<0.010$ & $<0.010$ \\
\hline
\end{tabular}


Table S5B. Concentrations ( $\mu \mathrm{g} \mathrm{L}^{-1}$ ) of carbonyl compounds in Plant B.

\begin{tabular}{|c|c|c|c|c|c|}
\hline Plant B - 2018 & WW Eff & $\mathrm{O}_{3} \mathrm{Eff}$ & MF Eff & $\begin{array}{l}\text { RO } \\
\text { Permeate }\end{array}$ & $\begin{array}{l}\mathrm{UV} / \mathrm{H}_{2} \mathrm{O}_{2} \\
\mathrm{Eff}\end{array}$ \\
\hline Formaldehyde & 7.43 & 115 & 111 & 82.9 & 55.3 \\
\hline Acetaldehyde & 11.7 & 105 & 102 & 32.8 & 21.4 \\
\hline Acetone & 11.1 & 35.3 & 31.6 & 16.3 & 14.2 \\
\hline Propionaldehyde & 1.30 & 14.2 & 13.4 & 5.07 & 1.56 \\
\hline Butyraldehyde & 0.800 & 6.37 & 5.47 & 1.94 & 0.476 \\
\hline Pentanal & 0.860 & 6.15 & 5.79 & 2.03 & 0.494 \\
\hline Hexanal & 0.530 & 12.8 & 11.4 & 2.83 & 0.783 \\
\hline Acrolein & 0.275 & 2.60 & 2.10 & 0.790 & 0.096 \\
\hline Crotonaldehyde & $<0.010$ & 1.30 & 0.980 & 0.354 & 0.026 \\
\hline 3-methyl-crotonaldehyde & $<0.010$ & 0.303 & 0.290 & 0.089 & $<0.010$ \\
\hline Trans-2-pentenal & $<0.010$ & 0.238 & 0.234 & 0.063 & $<0.010$ \\
\hline 2,4-hexadienal & $<0.010$ & $<0.010$ & $<0.010$ & $<0.010$ & $<0.010$ \\
\hline Trans-2-hexenal & $<0.010$ & 0.187 & 0.183 & 0.041 & $<0.010$ \\
\hline Trans-2-heptenal & $<0.010$ & $<0.010$ & $<0.010$ & $<0.010$ & $<0.010$ \\
\hline Plant B - 2019 & WW Eff & $\mathrm{O}_{3} \mathrm{Eff}$ & MF Eff & $\begin{array}{l}\text { RO } \\
\text { Permeate }\end{array}$ & $\begin{array}{l}\mathrm{UV} / \mathrm{H}_{2} \mathrm{O}_{2} \\
\mathrm{Eff}\end{array}$ \\
\hline Formaldehyde & 8.77 & 99.3 & 97.8 & 65.8 & 43.3 \\
\hline Acetaldehyde & 13.2 & 115 & 108 & 29.9 & 18.6 \\
\hline Acetone & 8.51 & 39.0 & 36.6 & 17.1 & 15.4 \\
\hline Propionaldehyde & 2.40 & 18.6 & 17.4 & 6.53 & 2.42 \\
\hline Butyraldehyde & 0.610 & 7.73 & 6.55 & 2.33 & 0.633 \\
\hline Pentanal & 0.520 & 7.82 & 7.27 & 1.91 & 0.466 \\
\hline Hexanal & 0.510 & 16.7 & 14.8 & 4.72 & 1.47 \\
\hline Acrolein & 0.178 & 4.69 & 5.04 & 1.98 & 0.201 \\
\hline Crotonaldehyde & $<0.010$ & 1.10 & 0.870 & 0.272 & 0.058 \\
\hline 3-methyl-crotonaldehyde & $<0.010$ & 0.283 & 0.279 & 0.073 & $<0.010$ \\
\hline Trans-2-pentenal & $<0.010$ & 0.291 & 0.154 & 0.042 & $<0.010$ \\
\hline 2,4-hexadienal & $<0.010$ & $<0.010$ & $<0.010$ & $<0.010$ & $<0.010$ \\
\hline Trans-2-hexenal & $<0.010$ & 0.205 & 0.198 & 0.046 & $<0.010$ \\
\hline Trans-2-heptenal & $<0.010$ & $<0.010$ & $<0.010$ & $<0.010$ & $<0.010$ \\
\hline
\end{tabular}


Table S5C. Concentrations ( $\mu \mathrm{g} \mathrm{L}^{-1}$ ) of carbonyl compounds in Plant C.

\begin{tabular}{|c|c|c|c|c|c|}
\hline Plant C - 2018 & WW Eff & MF Feed & MF Eff & $\begin{array}{l}\text { RO } \\
\text { Permeate }\end{array}$ & $\mathrm{UV} / \mathrm{H}_{2} \mathrm{O}_{2} \mathrm{Eff}$ \\
\hline Formaldehyde & 7.18 & 36.1 & 32.4 & 22.5 & 13.0 \\
\hline Acetaldehyde & 6.30 & 35.3 & 39.9 & 27.3 & 15.1 \\
\hline Acetone & 3.70 & 10.1 & 9.30 & 6.05 & 5.72 \\
\hline Propionaldehyde & 0.300 & 3.05 & 3.01 & 1.75 & 0.460 \\
\hline Butyraldehyde & 0.360 & 3.16 & 3.14 & 1.74 & 0.396 \\
\hline Pentanal & 0.250 & 2.40 & 2.20 & 1.04 & 0.220 \\
\hline Hexanal & 0.490 & 2.94 & 2.78 & 0.953 & 0.360 \\
\hline Acrolein & 0.500 & 0.900 & 0.890 & 0.500 & 0.068 \\
\hline Crotonaldehyde & $<0.010$ & 0.052 & 0.048 & 0.026 & $<0.010$ \\
\hline 3-methyl-crotonaldehyde & $<0.010$ & $<0.010$ & $<0.010$ & $<0.010$ & $<0.010$ \\
\hline Trans-2-pentenal & $<0.010$ & 0.024 & 0.022 & $<0.010$ & $<0.010$ \\
\hline 2,4-hexadienal & $<0.010$ & $<0.010$ & $<0.010$ & $<0.010$ & $<0.010$ \\
\hline Trans-2-hexenal & $<0.010$ & $<0.010$ & $<0.010$ & $<0.010$ & $<0.010$ \\
\hline Trans-2-heptenal & $<0.010$ & $<0.010$ & $<0.010$ & $<0.010$ & $<0.010$ \\
\hline Plant C - 2019 & WW Eff & MF Feed & MF Eff & $\begin{array}{l}\text { RO } \\
\text { Permeate }\end{array}$ & $\mathrm{UV} / \mathrm{H}_{2} \mathrm{O}_{2} \mathrm{Eff}$ \\
\hline Formaldehyde & 7.22 & 33.1 & 33.6 & 23.0 & 14.0 \\
\hline Acetaldehyde & 7.10 & 32.7 & 33.1 & 23.0 & 14.5 \\
\hline Acetone & 4.94 & 5.96 & 5.76 & 3.89 & 3.71 \\
\hline Propionaldehyde & 0.426 & 2.07 & 2.03 & 1.36 & 0.324 \\
\hline Butyraldehyde & 0.432 & 2.50 & 2.40 & 1.20 & 0.277 \\
\hline Pentanal & 0.392 & 2.14 & 2.01 & 1.09 & 0.217 \\
\hline Hexanal & 0.409 & 2.79 & 1.67 & 0.689 & 0.240 \\
\hline Acrolein & 0.620 & 0.608 & 0.639 & 0.418 & 0.034 \\
\hline Crotonaldehyde & $<0.010$ & 0.061 & 0.071 & 0.026 & $<0.010$ \\
\hline 3-methyl-crotonaldehyde & $<0.010$ & $<0.010$ & $<0.010$ & $<0.010$ & $<0.010$ \\
\hline Trans-2-pentenal & $<0.010$ & 0.037 & 0.031 & $<0.010$ & $<0.010$ \\
\hline 2,4-hexadienal & $<0.010$ & $<0.010$ & $<0.010$ & $<0.010$ & $<0.010$ \\
\hline Trans-2-hexenal & $<0.010$ & $<0.010$ & $<0.010$ & $<0.010$ & $<0.010$ \\
\hline Trans-2-heptenal & $<0.010$ & $<0.010$ & $<0.010$ & $<0.010$ & $<0.010$ \\
\hline
\end{tabular}


Table S5D. Concentrations ( $\mu \mathrm{g} \mathrm{L} \mathrm{L}^{-1}$ ) of carbonyl compounds in Plant D.

\begin{tabular}{|c|c|c|c|c|c|}
\hline Plant D - 2018 & WW Eff & MF Feed & MF Eff & $\begin{array}{l}\text { RO } \\
\text { Permeate }\end{array}$ & $\mathrm{UV} / \mathrm{H}_{2} \mathrm{O}_{2} \mathrm{Eff}$ \\
\hline Formaldehyde & 2.20 & 20.3 & 17.3 & 10.9 & 6.57 \\
\hline Acetaldehyde & 8.00 & 39.6 & 38.4 & 21.3 & 10.4 \\
\hline Acetone & 6.78 & 11.5 & 11.3 & 6.97 & 5.97 \\
\hline Propionaldehyde & 0.504 & 3.20 & 3.17 & 1.23 & 0.349 \\
\hline Butyraldehyde & 0.603 & 3.80 & 2.90 & 1.03 & 0.246 \\
\hline Pentanal & 0.611 & 3.00 & 2.80 & 0.82 & 0.105 \\
\hline Hexanal & 0.781 & 2.90 & 2.80 & 0.81 & 0.232 \\
\hline Acrolein & $<0.010$ & 1.45 & 1.43 & 0.81 & 0.057 \\
\hline Crotonaldehyde & $<0.010$ & 0.280 & 0.270 & 0.098 & $<0.010$ \\
\hline 3-methyl-crotonaldehyde & $<0.010$ & 0.103 & 0.029 & $<0.010$ & $<0.010$ \\
\hline Trans-2-pentenal & $<0.010$ & 0.057 & 0.060 & $<0.010$ & $<0.010$ \\
\hline 2,4-hexadienal & $<0.010$ & $<0.010$ & $<0.010$ & $<0.010$ & $<0.010$ \\
\hline Trans-2-hexenal & $<0.010$ & $<0.010$ & $<0.010$ & $<0.010$ & $<0.010$ \\
\hline Trans-2-heptenal & $<0.010$ & $<0.010$ & $<0.010$ & $<0.010$ & $<0.010$ \\
\hline Plant D - 2019 & WW Eff & MF Feed & MF Eff & $\begin{array}{l}\text { RO } \\
\text { Permeate }\end{array}$ & $\mathrm{UV} / \mathrm{H}_{2} \mathrm{O}_{2} \mathrm{Eff}$ \\
\hline Formaldehyde & 1.84 & 19.3 & 18.9 & 12.6 & 9.92 \\
\hline Acetaldehyde & 5.76 & 41.6 & 39.0 & 19.1 & 9.30 \\
\hline Acetone & 4.91 & 10.9 & 10.7 & 7.17 & 6.01 \\
\hline Propionaldehyde & 0.533 & 5.12 & 5.01 & 2.16 & 0.706 \\
\hline Butyraldehyde & 0.808 & 3.75 & 3.57 & 1.27 & 0.294 \\
\hline Pentanal & 0.780 & 1.63 & 1.57 & 0.580 & 0.101 \\
\hline Hexanal & 0.795 & 4.31 & 3.58 & 0.980 & 0.267 \\
\hline Acrolein & $<0.010$ & 1.89 & 1.78 & 0.910 & 0.059 \\
\hline Crotonaldehyde & $<0.010$ & 0.530 & 0.510 & 0.191 & $<0.010$ \\
\hline 3-methyl-crotonaldehyde & $<0.010$ & 0.112 & 0.035 & $<0.010$ & $<0.010$ \\
\hline Trans-2-pentenal & $<0.010$ & 0.043 & 0.041 & $<0.010$ & $<0.010$ \\
\hline 2,4-hexadienal & $<0.010$ & $<0.010$ & $<0.010$ & $<0.010$ & $<0.010$ \\
\hline Trans-2-hexenal & $<0.010$ & $<0.010$ & $<0.010$ & $<0.010$ & $<0.010$ \\
\hline Trans-2-heptenal & $<0.010$ & $<0.010$ & $<0.010$ & $<0.010$ & $<0.010$ \\
\hline
\end{tabular}


Table S5E. Concentrations $\left(\mu \mathrm{g} \mathrm{L}^{-1}\right)$ of carbonyl compounds in Plant E.

\begin{tabular}{|c|c|c|c|c|c|c|}
\hline Plant E - 2018 & WW Eff & $\mathrm{O}_{3} \mathrm{Eff}$ & BAC Eff & UF Eff & $\begin{array}{l}\text { RO } \\
\text { Permeate }\end{array}$ & $\begin{array}{l}\mathrm{UV} / \mathrm{HOCl} \\
\mathrm{Eff}\end{array}$ \\
\hline Formaldehyde & 1.82 & 73.1 & 2.78 & 2.18 & 1.45 & 1.01 \\
\hline Acetaldehyde & 10.9 & 106 & 12.0 & 10.3 & 6.48 & 4.66 \\
\hline Acetone & 4.53 & 32.8 & 6.95 & 6.86 & 4.41 & 3.91 \\
\hline Propionaldehyde & 0.135 & 11.7 & 0.566 & 0.502 & 0.270 & $<0.050$ \\
\hline Butyraldehyde & 0.135 & 5.76 & 0.538 & 0.509 & 0.265 & $<0.050$ \\
\hline Pentanal & 0.452 & 5.17 & 0.317 & 0.311 & 0.158 & $<0.050$ \\
\hline Hexanal & 0.335 & 5.57 & 0.658 & 0.627 & 0.270 & $<0.050$ \\
\hline Acrolein & 0.020 & 2.26 & 0.139 & 0.134 & 0.075 & $<0.010$ \\
\hline Crotonaldehyde & $<0.010$ & 0.319 & $<0.010$ & $<0.010$ & $<0.010$ & $<0.010$ \\
\hline 3-methyl-crotonaldehyde & $<0.010$ & 0.139 & $<0.010$ & $<0.010$ & $<0.010$ & $<0.010$ \\
\hline Trans-2-pentenal & $<0.010$ & 0.221 & $<0.010$ & $<0.010$ & $<0.010$ & $<0.010$ \\
\hline 2,4-hexadienal & $<0.010$ & $<0.010$ & $<0.010$ & $<0.010$ & $<0.010$ & $<0.010$ \\
\hline Trans-2-hexenal & $<0.010$ & 0.104 & $<0.010$ & $<0.010$ & $<0.010$ & $<0.010$ \\
\hline Trans-2-heptenal & $<0.010$ & $<0.010$ & $<0.010$ & $<0.010$ & $<0.010$ & $<0.010$ \\
\hline Plant E - 2019 & WW Eff & $\mathrm{O}_{3} \mathrm{Eff}$ & BAC Eff & UF Eff & $\begin{array}{l}\text { RO } \\
\text { Permeate }\end{array}$ & $\begin{array}{l}\mathrm{UV} / \mathrm{HOCl} \\
\mathrm{Eff}\end{array}$ \\
\hline Formaldehyde & 0.770 & 81.0 & 2.13 & 3.12 & 1.99 & 1.42 \\
\hline Acetaldehyde & 5.17 & 111 & 11.7 & 10.3 & 5.11 & 3.71 \\
\hline Acetone & 7.95 & 43.8 & 5.75 & 4.99 & 3.02 & 2.72 \\
\hline Propionaldehyde & 0.153 & 10.2 & 0.554 & 0.559 & 0.310 & $<0.025$ \\
\hline Butyraldehyde & 0.315 & 5.66 & 0.498 & 0.488 & 0.249 & $<0.025$ \\
\hline Pentanal & 0.206 & 5.21 & 0.426 & 0.422 & 0.200 & $<0.025$ \\
\hline Hexanal & 0.301 & 5.46 & 0.553 & 0.403 & 0.210 & $<0.025$ \\
\hline Acrolein & 0.100 & 2.06 & 0.195 & 0.159 & 0.078 & $<0.010$ \\
\hline Crotonaldehyde & $<0.010$ & 0.313 & $<0.010$ & $<0.010$ & $<0.010$ & $<0.010$ \\
\hline 3-methyl-crotonaldehyde & $<0.010$ & 0.147 & $<0.010$ & $<0.010$ & $<0.010$ & $<0.010$ \\
\hline Trans-2-pentenal & $<0.010$ & 0.228 & $<0.010$ & $<0.010$ & $<0.010$ & $<0.010$ \\
\hline 2,4-hexadienal & $<0.010$ & $<0.010$ & $<0.010$ & $<0.010$ & $<0.010$ & $<0.010$ \\
\hline Trans-2-hexenal & $<0.010$ & 0.110 & $<0.010$ & $<0.010$ & $<0.010$ & $<0.010$ \\
\hline Trans-2-heptenal & $<0.010$ & $<0.010$ & $<0.010$ & $<0.010$ & $<0.010$ & $<0.010$ \\
\hline
\end{tabular}


Table S5F. Concentrations $\left(\mu \mathrm{g} \mathrm{L}^{-1}\right)$ of carbonyl compounds in Plant F.

\begin{tabular}{|c|c|c|c|c|c|}
\hline Plant F - 2018 & WW Eff & $\mathrm{O}_{3} \mathrm{Eff}$ & BAC Eff & GAC Eff & UV Eff \\
\hline Formaldehyde & 1.74 & 94.3 & 4.01 & 3.56 & 31.0 \\
\hline Acetaldehyde & 4.92 & 104 & 5.12 & 2.39 & 10.5 \\
\hline Acetone & 5.47 & 20.6 & 4.17 & 3.60 & 4.46 \\
\hline Propionaldehyde & 1.10 & 15.6 & 1.71 & 0.421 & 1.22 \\
\hline Butyraldehyde & 0.285 & 5.22 & 0.244 & 0.213 & 0.126 \\
\hline Pentanal & 0.711 & 4.73 & 0.229 & 0.153 & 0.144 \\
\hline Hexanal & 0.239 & 3.99 & 0.161 & 0.144 & 0.109 \\
\hline Acrolein & $<0.010$ & 2.42 & 0.244 & 0.223 & 0.243 \\
\hline Crotonaldehyde & $<0.010$ & 0.484 & $<0.010$ & $<0.010$ & 0.020 \\
\hline 3-methyl-crotonaldehyde & $<0.010$ & 0.102 & $<0.010$ & $<0.010$ & $<0.010$ \\
\hline Trans-2-pentenal & $<0.010$ & 0.225 & $<0.010$ & $<0.010$ & $<0.010$ \\
\hline 2,4-hexadienal & $<0.010$ & $<0.010$ & $<0.010$ & $<0.010$ & $<0.010$ \\
\hline Trans-2-hexenal & $<0.010$ & 0.106 & $<0.010$ & $<0.010$ & $<0.010$ \\
\hline Trans-2-heptenal & $<0.010$ & $<0.010$ & $<0.010$ & $<0.010$ & $<0.010$ \\
\hline Plant F - 2019 & WW Eff & $\mathrm{O}_{3} \mathrm{Eff}$ & BAC Eff & GAC Eff & UV Eff \\
\hline Formaldehyde & 3.69 & 74.0 & 5.42 & 3.02 & 31.4 \\
\hline Acetaldehyde & 6.48 & 83.4 & 7.19 & 3.26 & 12.5 \\
\hline Acetone & 6.17 & 16.6 & 4.16 & 4.52 & 5.72 \\
\hline Propionaldehyde & 1.25 & 19.2 & 1.52 & 0.678 & 1.40 \\
\hline Butyraldehyde & 0.354 & 4.89 & 0.157 & 0.181 & 0.534 \\
\hline Pentanal & 0.757 & 3.89 & 0.162 & 0.204 & 0.430 \\
\hline Hexanal & 0.511 & 3.34 & 0.128 & 0.212 & 0.307 \\
\hline Acrolein & $<0.010$ & 2.32 & 0.256 & 0.239 & 0.258 \\
\hline Crotonaldehyde & $<0.010$ & 0.497 & $<0.010$ & $<0.010$ & 0.035 \\
\hline 3-methyl-crotonaldehyde & $<0.010$ & 0.111 & $<0.010$ & $<0.010$ & $<0.010$ \\
\hline Trans-2-pentenal & $<0.010$ & 0.259 & $<0.010$ & $<0.010$ & $<0.010$ \\
\hline 2,4-hexadienal & $<0.010$ & $<0.010$ & $<0.010$ & $<0.010$ & $<0.010$ \\
\hline Trans-2-hexenal & $<0.010$ & 0.110 & $<0.010$ & $<0.010$ & $<0.010$ \\
\hline Trans-2-heptenal & $<0.010$ & $<0.010$ & $<0.010$ & $<0.010$ & $<0.010$ \\
\hline
\end{tabular}


Table S5G. Concentrations ( $\mu \mathrm{g} \mathrm{L}^{-1}$ ) of carbonyl compounds in Plant G.

\begin{tabular}{|c|c|c|c|c|c|}
\hline Plant G - 2019A & Raw Water & Settled Eff & $\mathrm{O}_{3} \mathrm{Eff}$ & $\begin{array}{l}\text { Filtration } \\
\text { Eff }\end{array}$ & $\begin{array}{l}\text { Disinfection } \\
\text { Eff }\end{array}$ \\
\hline Formaldehyde & 3.17 & 2.19 & 19.7 & 18.0 & 16.2 \\
\hline Acetaldehyde & 2.50 & 3.59 & 20.4 & 19.2 & 18.9 \\
\hline Acetone & 2.20 & 2.31 & 10.9 & 10.0 & 10.4 \\
\hline Propionaldehyde & 0.349 & 0.799 & 2.14 & 0.716 & 0.884 \\
\hline Butyraldehyde & 0.238 & 0.137 & 0.463 & 0.207 & 0.217 \\
\hline Pentanal & $<0.050$ & $<0.050$ & 0.304 & 0.205 & 0.158 \\
\hline Hexanal & $<0.050$ & $<0.050$ & 0.556 & 0.139 & 0.192 \\
\hline Acrolein & $<0.010$ & $<0.010$ & 0.259 & 0.272 & 0.206 \\
\hline Crotonaldehyde & $<0.010$ & $<0.010$ & 0.147 & 0.076 & 0.026 \\
\hline 3-methyl-crotonaldehyde & $<0.010$ & $<0.010$ & $<0.010$ & $<0.010$ & $<0.010$ \\
\hline Trans-2-pentenal & $<0.010$ & $<0.010$ & $<0.010$ & $<0.010$ & $<0.010$ \\
\hline 2,4-hexadienal & $<0.010$ & $<0.010$ & $<0.010$ & $<0.010$ & $<0.010$ \\
\hline Trans-2-hexenal & $<0.010$ & $<0.010$ & $<0.010$ & $<0.010$ & $<0.010$ \\
\hline Trans-2-heptenal & $<0.010$ & $<0.010$ & $<0.010$ & $<0.010$ & $<0.010$ \\
\hline Plant G - 2019B & Raw Water & Settled Eff & $\mathrm{O}_{3} \mathrm{Eff}$ & $\begin{array}{l}\text { Filtration } \\
\text { Eff }\end{array}$ & $\begin{array}{l}\text { Disinfection } \\
\text { Eff }\end{array}$ \\
\hline Formaldehyde & 2.54 & 2.00 & 20.0 & 20.7 & 21.7 \\
\hline Acetaldehyde & 3.19 & 2.87 & 25.8 & 24.5 & 26.3 \\
\hline Acetone & 2.83 & 2.95 & 12.1 & 11.0 & 12.7 \\
\hline Propionaldehyde & 0.298 & 0.742 & 2.52 & 0.732 & 0.947 \\
\hline Butyraldehyde & 0.135 & 0.125 & 0.375 & 0.170 & 0.163 \\
\hline Pentanal & $<0.050$ & $<0.050$ & 0.301 & 0.296 & 0.201 \\
\hline Hexanal & $<0.050$ & $<0.050$ & 0.485 & 0.163 & 0.192 \\
\hline Acrolein & $<0.010$ & $<0.010$ & 0.349 & 0.327 & 0.333 \\
\hline Crotonaldehyde & $<0.010$ & $<0.010$ & 0.170 & 0.084 & 0.031 \\
\hline 3-methyl-crotonaldehyde & $<0.010$ & $<0.010$ & $<0.010$ & $<0.010$ & $<0.010$ \\
\hline Trans-2-pentenal & $<0.010$ & $<0.010$ & $<0.010$ & $<0.010$ & $<0.010$ \\
\hline 2,4-hexadienal & $<0.010$ & $<0.010$ & $<0.010$ & $<0.010$ & $<0.010$ \\
\hline Trans-2-hexenal & $<0.010$ & $<0.010$ & $<0.010$ & $<0.010$ & $<0.010$ \\
\hline Trans-2-heptenal & $<0.010$ & $<0.010$ & $<0.010$ & $<0.010$ & $<0.010$ \\
\hline
\end{tabular}


Figures S7A-G. Graphs of individual sampling events from 2018 and 2019 for Plants A-G.
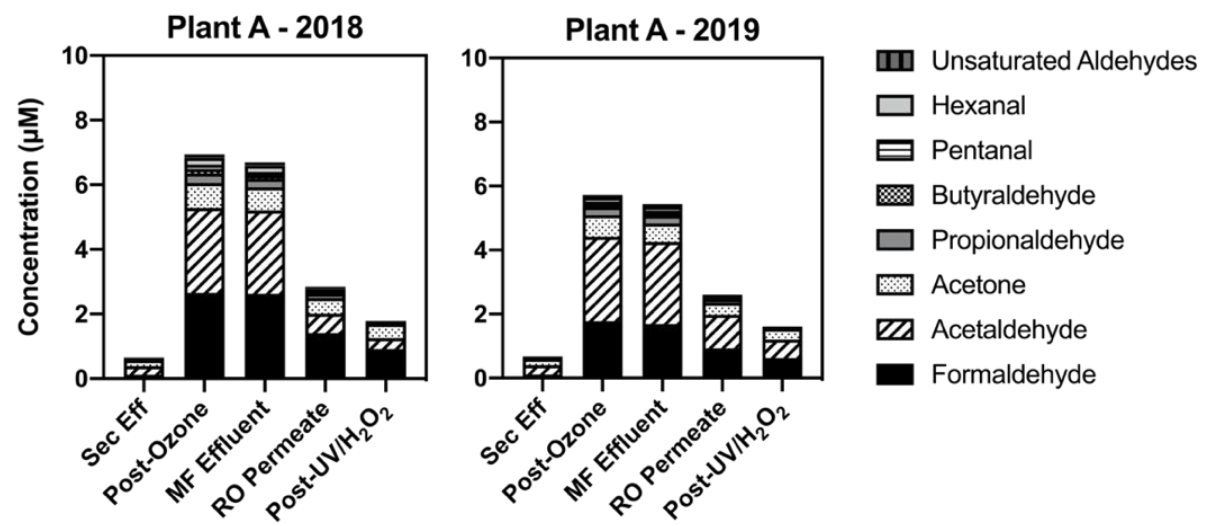

Figure S7A. Graphical representation (in $\mu \mathrm{M}$ ) of data in Table A4A from individual sampling events at Plant A in 2018 and 2019.
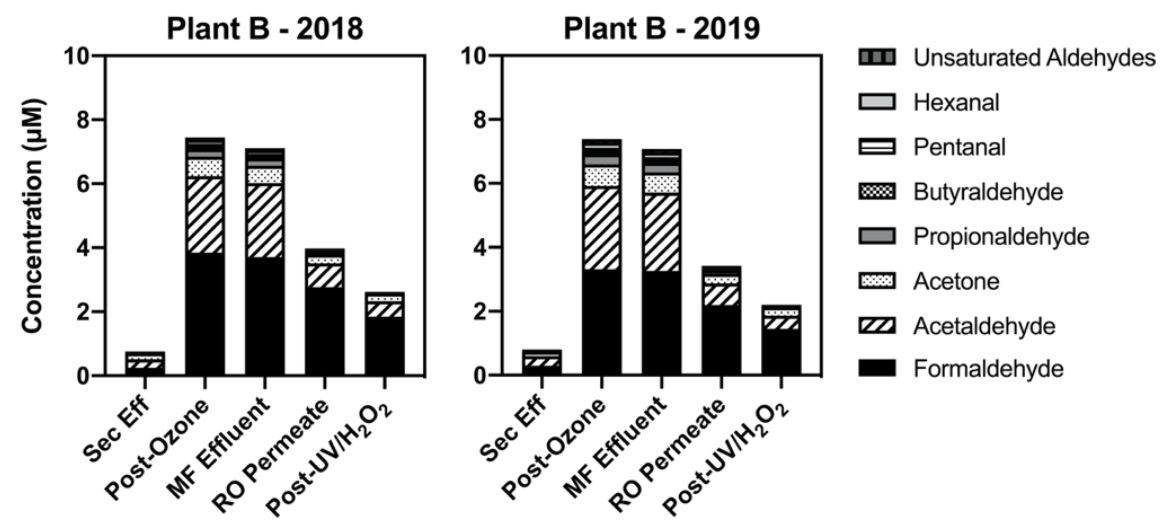

Figure S7B. Graphical representation (in $\mu \mathrm{M}$ ) of data in Table A4B from individual sampling events at Plant B in 2018 and 2019. 

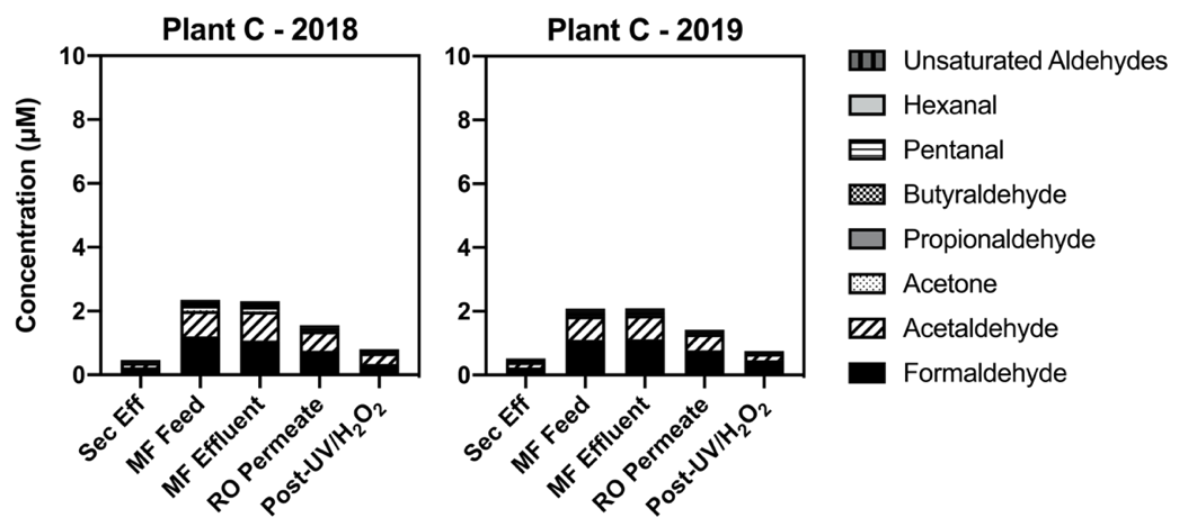

Figure S7C. Graphical representation (in $\mu \mathrm{M}$ ) of data in Table A4C from individual sampling events at Plant C in 2018 and 2019.
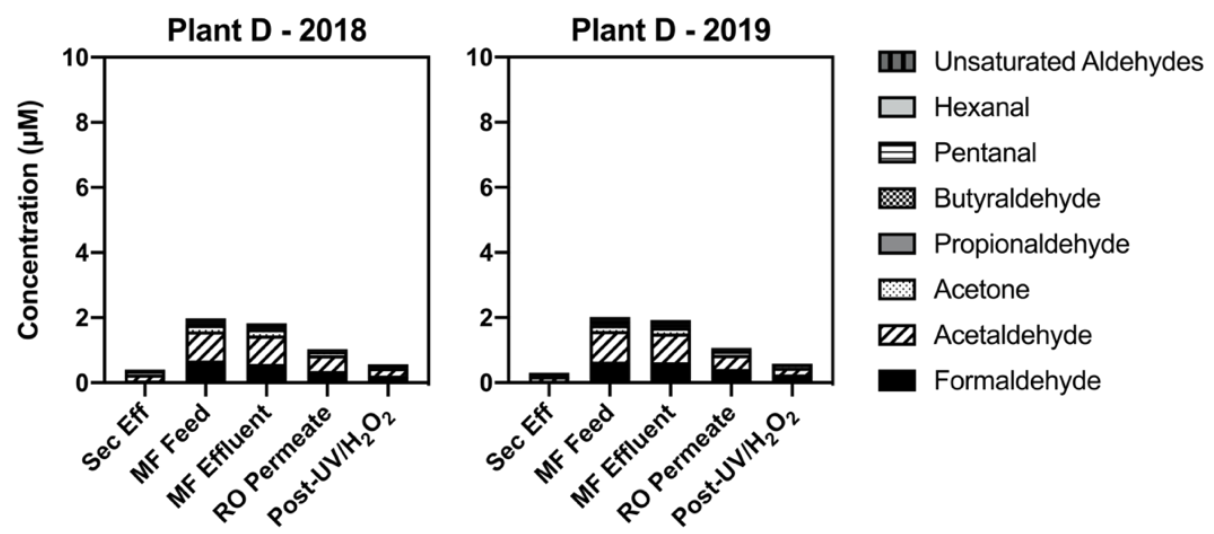

Figure S7D. Graphical representation (in $\mu \mathrm{M}$ ) of data in Table A4D from individual sampling events at Plant D in 2018 and 2019.
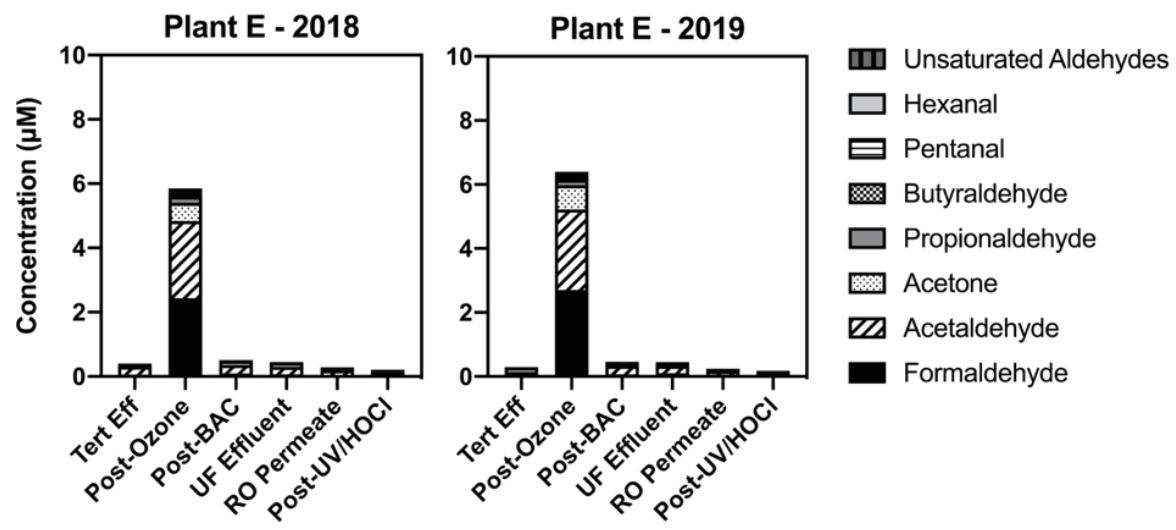

Figure S7E. Graphical representation (in $\mu \mathrm{M}$ ) of data in Table A4E from individual sampling events at Plant E in 2018 and 2019. 

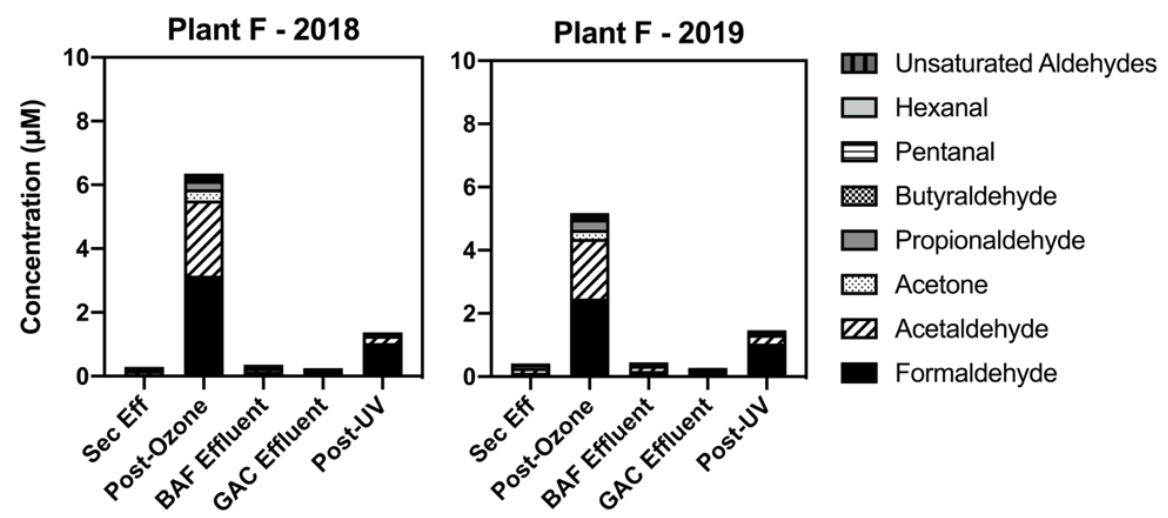

Figure S7F. Graphical representation (in $\mu \mathrm{M}$ ) of data in Table A4F from individual sampling events at Plant F in 2018 and 2019.
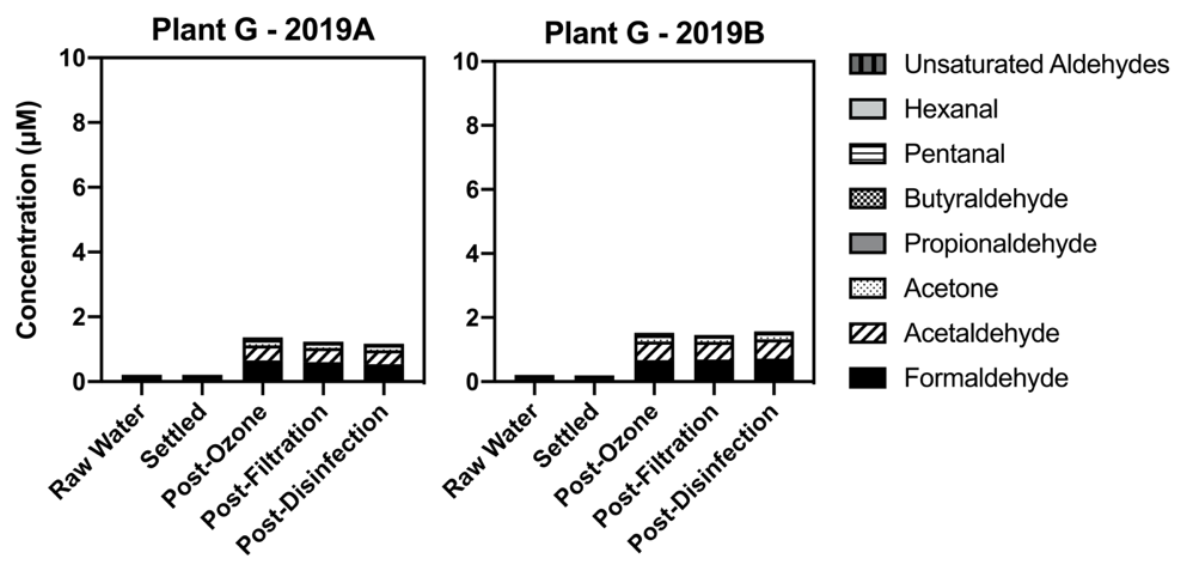

Figure S7G. Graphical representation (in $\mu \mathrm{M}$ ) of data in Table A4G from individual sampling events at Plant G in 2019. 
Table S6. Chlorination experiment results compared to MFF samples. Concentrations of carbonyl compounds in Plant D from MF-feed grab samples (left) and after laboratory-based chlorination of Plant D influent (wastewater effluent, right).

\begin{tabular}{|c|c|c|c|c|}
\hline \multirow{3}{*}{ Compound } & \multicolumn{4}{|c|}{ Concentration, Plant $D\left(\mu \mathrm{g} \mathrm{L}^{-1}\right)$} \\
\hline & \multicolumn{2}{|c|}{ MF Feed-Grab } & \multicolumn{2}{|c|}{ INF+NaOCl spike } \\
\hline & $\mu g \mathrm{~L}^{-1}$ & $\mu \mathrm{M}$ & $\mu g L^{-1}$ & $\mu \mathrm{M}$ \\
\hline Formaldehyde & 19.3 & 0.640 & 18.5 & 0.617 \\
\hline Acetaldehyde & 41.6 & 0.945 & 42.9 & 0.975 \\
\hline Acetone & 10.9 & 0.188 & 10.12 & 0.174 \\
\hline Propionaldehyde & 5.12 & 0.088 & 5.35 & 0.092 \\
\hline Butyraldehyde & 3.75 & 0.052 & 3.95 & 0.055 \\
\hline Pentanal & 1.63 & 0.019 & 1.49 & 0.017 \\
\hline Hexanal & 4.31 & 0.043 & 4.73 & 0.047 \\
\hline Acrolein & 1.89 & 0.034 & 1.99 & 0.036 \\
\hline Crotonaldehyde & 0.53 & 7.57E-03 & 0.5 & 7.14E-03 \\
\hline 3-methy crotonaldehyde & 0.11 & $1.31 \mathrm{E}-03$ & 0.13 & $1.55 \mathrm{E}-03$ \\
\hline Trans-2-pentenal & 0.043 & $5.12 \mathrm{E}-04$ & 0.047 & $5.60 \mathrm{E}-04$ \\
\hline 2,4-hexadienal & $<0.005$ & $<5 \mathrm{E}-05$ & 0.005 & $<5 \mathrm{E}-05$ \\
\hline Trans-2-hexenal & $<0.005$ & $<5 \mathrm{E}-05$ & 0.005 & $<5 \mathrm{E}-05$ \\
\hline Trans-2-heptenal & $<0.005$ & $<5 E-05$ & 0.005 & $<5 \mathrm{E}-05$ \\
\hline
\end{tabular}


Table S7. Reaction rate constants $\left(k_{\mathrm{HO}} \cdot\right)$ between select carbonyl compounds and hydroxyl radical $\left(\mathrm{HO}^{\circ}\right)$. Corresponding references in last column.

\begin{tabular}{|c|c|c|c|c|}
\hline Compound & $\boldsymbol{k}_{\text {HO }}\left(\mathbf{M}^{-1} \mathbf{s}^{-1}\right)$ & log $\boldsymbol{k}_{\text {HO }}$ & \% Remaining & $\begin{array}{c}\text { Reference for } \\
\boldsymbol{k}_{\text {HO }}\end{array}$ \\
\hline Formaldehyde & $1.0 \times 10^{9}$ & 9.0 & 66 & 3 \\
\hline Acetaldehyde & $7.3 \times 10^{8}$ & 8.9 & 72 & 3 \\
\hline Acetone & $1.3 \times 10^{8}$ & 8.1 & 95 & 3 \\
\hline Propionaldehyde & $2.9 \times 10^{9}$ & 9.5 & 27 & 4 \\
\hline Butyraldehyde & $3.8 \times 10^{9}$ & 9.6 & 19 & 4 \\
\hline Pentanal & $3.9 \times 10^{9}$ & 9.6 & 19 & 5 \\
\hline Hexanal & $2.5 \times 10^{9}$ & 9.4 & 35 & 3 \\
\hline Acrolein & $7.0 \times 10^{9}$ & 9.8 & 7.5 & 3 \\
\hline Crotonaldehyde & $5.8 \times 10^{9}$ & 9.8 & 7.5 & \\
\hline
\end{tabular}




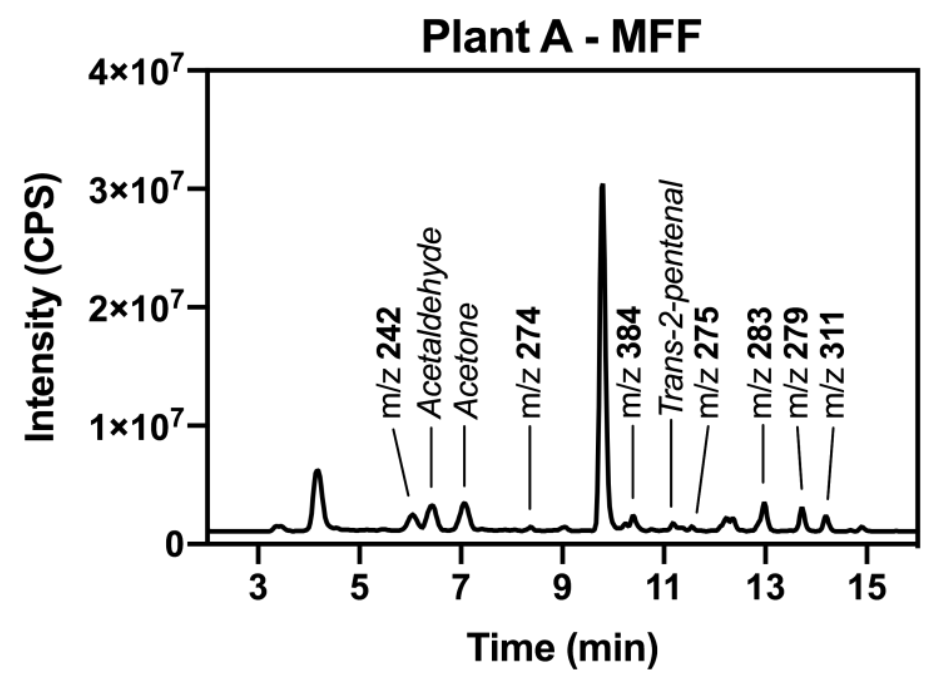

Figure S8. Plant A precursor ion scan (m/z 139) for TSH-adducts in the MF feed (MFF).

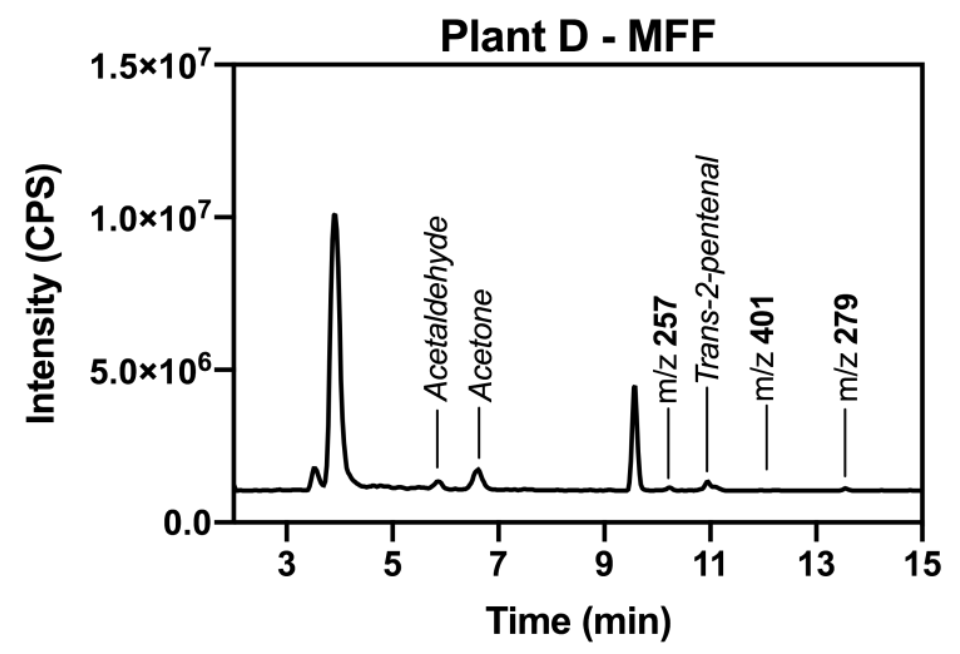

Figure S9. Plant D precursor ion scan (m/z 139) for TSH-adducts in the MF feed (MFF). 


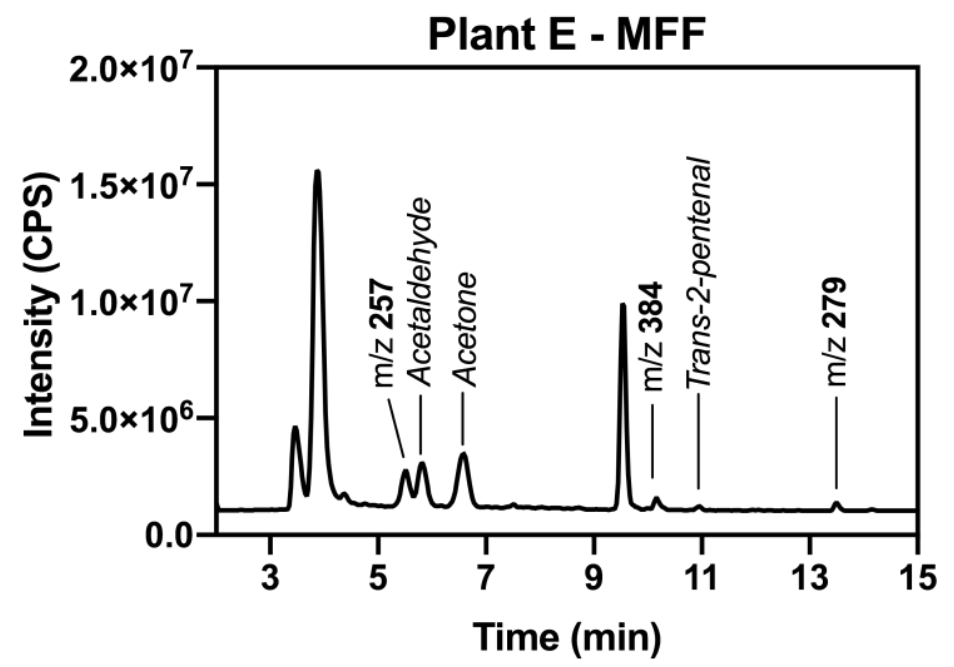

Figure S10. Plant E precursor ion scan (m/z 139) for TSH-adducts in the MF feed (MFF).

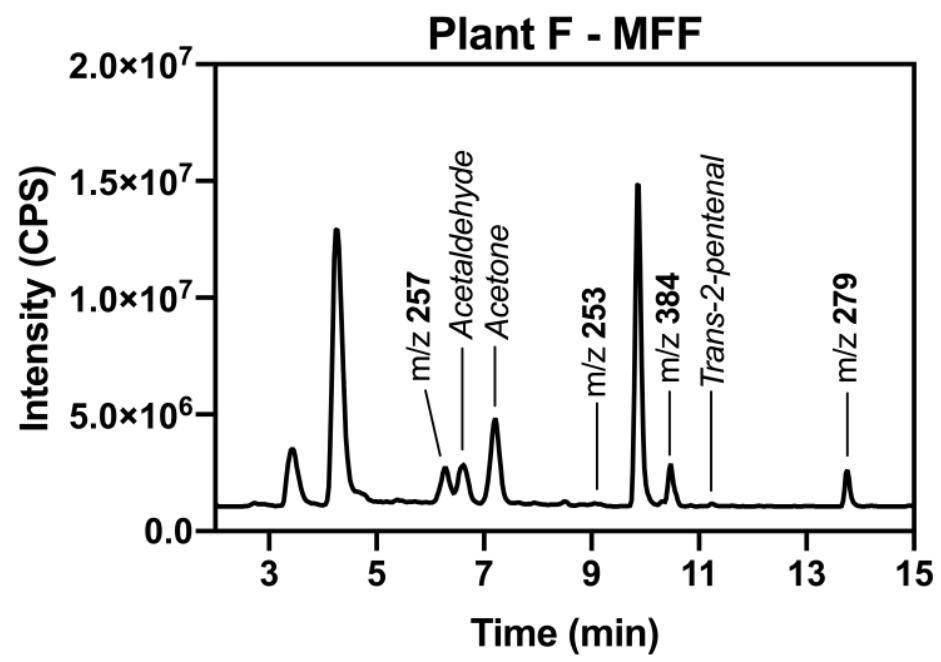

Figure S11. Plant F precursor ion scan (m/z 139) for TSH-adducts in the MF feed (MFF). 
Table S8. Identified and unidentified peaks from TSH precursor ion scans (m/z 139) of MF feed samples from Plants A, D, E, and G. "RT" = retention times on corresponding chromatograms (Figures S8-S11).

\begin{tabular}{|c|c|c|c|c|c|c|c|c|c|c|}
\hline \multirow{3}{*}{ Facility } & \multicolumn{10}{|c|}{ Compound } \\
\hline & \multicolumn{6}{|c|}{ Targeted } & \multicolumn{4}{|c|}{ Unidentified } \\
\hline & Name & RT & $\begin{array}{c}m / z \\
(+)\end{array}$ & $\begin{array}{c}\mathbf{m} / \mathbf{z} \\
(-\mathrm{TSH})\end{array}$ & Peak Area & $\begin{array}{c}\text { Conc. } \\
\left(\mu \mathrm{g} \mathrm{L}^{-1}\right)\end{array}$ & RT & $\begin{array}{c}m / z \\
(+)\end{array}$ & $\begin{array}{c}\mathrm{m} / \mathbf{z} \\
(-\mathrm{TSH})\end{array}$ & Peak Area \\
\hline \multirow{7}{*}{ Plant A } & Acetaldehyde & 7.08 & 213 & 44 & 29329391 & 115 & 6.06 & 242 & 73 & 8034424 \\
\hline & Acetone & 7.92 & 227 & 58 & 472098 & 44.7 & 8.38 & 274 & 105 & 497922 \\
\hline & Trans-2-pentenal & 11.20 & 253 & 84 & 2280718 & 0.180 & 10.41 & 384 & 215 & 4388992 \\
\hline & & & & & & & 11.56 & 275 & 106 & 2229162 \\
\hline & & & & & & & 12.99 & 283 & 114 & 15725934 \\
\hline & & & & & & & 13.75 & 279 & 110 & 8357823 \\
\hline & & & & & & & 14.18 & 311 & 142 & 8134835 \\
\hline \multirow{3}{*}{ Plant D } & Acetaldehyde & 6.63 & 213 & 44 & 7902520 & 39.6 & 10.97 & 257 & 88 & 2632421 \\
\hline & Acetone & 7.49 & 227 & 58 & 58420 & 38.4 & 11.97 & 401 & 232 & 24949 \\
\hline & Trans-2-pentenal & 10.97 & 253 & 84 & 221163 & 0.057 & 13.55 & 279 & 110 & 208570 \\
\hline \multirow{3}{*}{ Plant E } & Acetaldehyde & 6.57 & 213 & 44 & 31879685 & 106 & 5.52 & 257 & 88 & 4816948 \\
\hline & Acetone & 7.52 & 227 & 58 & 766841 & 32.8 & 10.16 & 384 & 215 & 1874177 \\
\hline & Trans-2-pentenal & 10.97 & 253 & 84 & 1089802 & 0.221 & 13.51 & 279 & 110 & 1153217 \\
\hline \multirow{4}{*}{ Plant F } & Acetaldehyde & 7.22 & 213 & 44 & 42393084 & 104 & 6.30 & 257 & 88 & 12079939 \\
\hline & Acetone & 8.00 & 227 & 58 & 478841 & 20.6 & 9.20 & 253 & 84 & 88278 \\
\hline & Trans-2-pentenal & 11.20 & 253 & 84 & 638402 & 0.225 & 10.49 & 384 & 215 & 6670678 \\
\hline & & & & & & & 13.75 & 279 & 110 & 6711216 \\
\hline
\end{tabular}



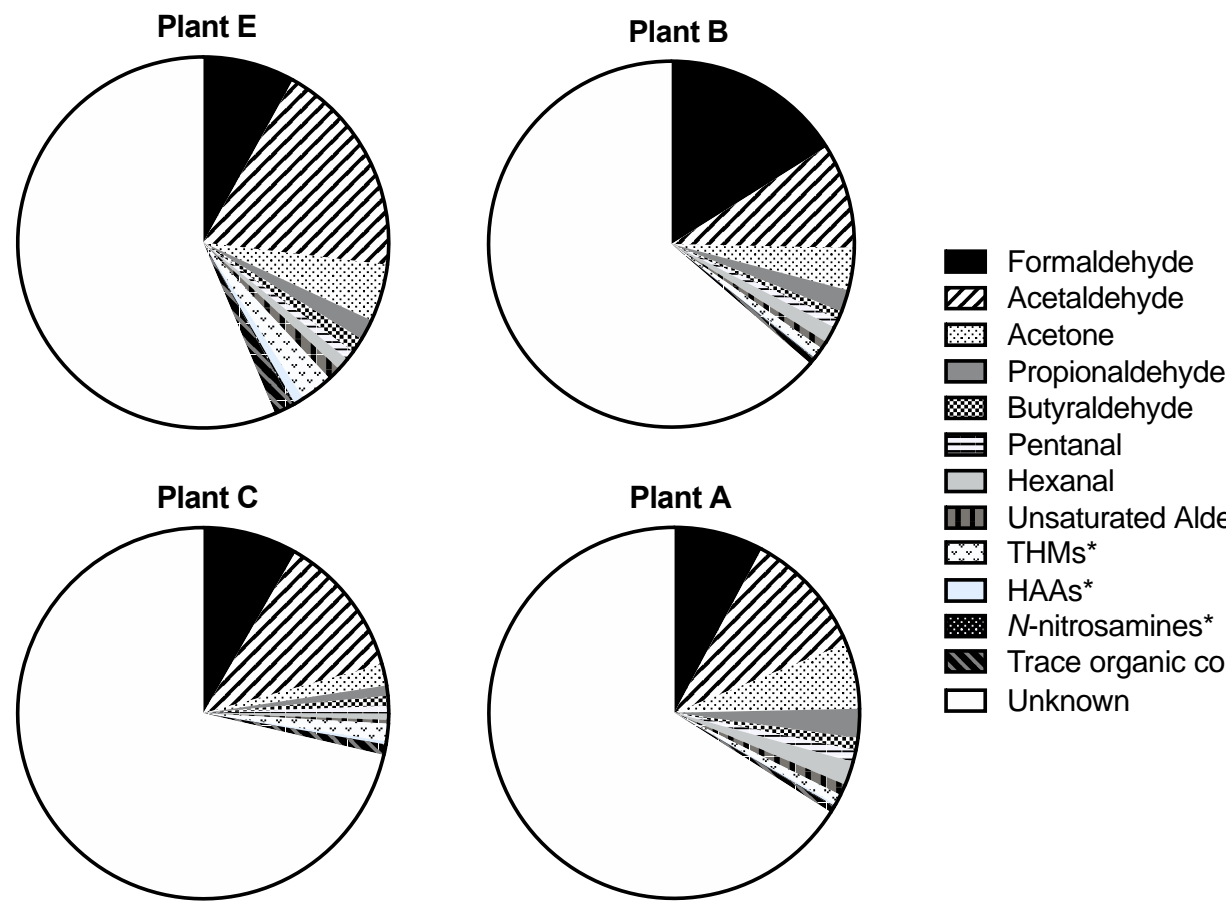
$\square$ Hexanal
Uा Unsaturated Aldehydes
THMs*
$\square$ HAAs* $^{*}$
N-nitrosamines ${ }^{*}$
NV Trace organic contaminants*
$\square$ Unknown

Figure S12. The contribution of carbonyl compounds and other chemicals to the total DOC concentration in RO permeate for Plants A, B, C, and E. *Values for trace organic contaminants, as well as nitrogenous and halogenated disinfection byproducts, were not generated during this work and were taken from elsewhere for similar treatment trains. ${ }^{6,7}$ 


\section{References}

(1) Rice, E. .; Baird, R.; Eaton, A. .; Clesceri, L. . 5310 Total Organic Carbon (TOC). Stand. Methods Exam. Water Wastewater 2005, No. 5310, 1-16.

(2) Hahnenstein, I.; Hasse, H.; Kreiter, C. G.; Maurer, G. 1H- and 13C-NMR-Spectroscopic Study of Chemical Equilibria in Solutions of Formaldehyde in Water, Deuterium Oxide, and Methanol. Ind. Eng. Chem. Res. 1994, 33 (4), 1022-1029.

(3) Buxton, G. V.; Greenstock, C. L.; Helman, W. P.; Ross, A. B. Critical Review of Rate Constants for Reactions of Hydrated Electrons, Hydrogen Atoms and Hydroxyl Radicals $\left(\cdot \mathrm{OH} / \mathrm{O}^{-}\right)$in Aqueous Solution. J. Phys. Chem. Ref. Data 1988, 17 (2), 513-886.

(4) Monod, A.; Poulain, L.; Grubert, S.; Voisin, D.; Wortham, H. Kinetics of OH-Initiated Oxidation of Oxygenated Organic Compounds in the Aqueous Phase: New Rate Constants, Structure-Activity Relationships and Atmospheric Implications. Atmos. Environ. 2005, 39 (40), 7667-7688.

(5) Jürgens, M.; Jacob, F.; Ekici, P.; Friess, A.; Parlar, H. Determination of Direct Photolysis Rate Constants and $\mathrm{OH}$ Radical Reactivity of Representative Odour Compounds in Brewery Broth Using a Continuous Flow-Stirred Photoreactor. Atmos. Environ. 2007, 41 (22), 4571-4584.

(6) Orange County Water District. Groundwater Replenishment System 2017 Annual Report; Irvine, CA, 2017.

(7) Zeng, T.; Plewa, M. J.; Mitch, W. A. N-Nitrosamines and Halogenated Disinfection Byproducts in U.S. Full Advanced Treatment Trains for Potable Reuse. Water Res. 2016, 101, 176-186. 